\title{
Decay Estimates for a Type of Fuzzy Viscoelastic Integro-Differential Model
}

\author{
Fengyun Zhang $\mathbb{D}^{1},{ }^{1}$ Funing Lin $\mathbb{D}^{2,3}$ Guangwang $\mathrm{Su}^{2,3}$ and Guangming $\mathrm{Xue}^{2,3}$ \\ ${ }^{1}$ Department of Mathematics, Jining University, Qufu 273155, China \\ ${ }^{2}$ School of Information and Statistics, Guangxi University of Finance and Economics, Nanning 530003, China \\ ${ }^{3}$ Guangxi Key Laboratory of Cross-border E-commerce Intelligent Information Processing, \\ Guangxi University of Finance and Economics, Nanning 530003, China
}

Correspondence should be addressed to Funing Lin; toplin518@126.com

Received 13 January 2021; Revised 6 February 2021; Accepted 22 February 2021; Published 15 March 2021

Academic Editor: Ahmed Mostafa Khalil

Copyright (c) 2021 Fengyun Zhang et al. This is an open access article distributed under the Creative Commons Attribution License, which permits unrestricted use, distribution, and reproduction in any medium, provided the original work is properly cited.

We consider a type of fuzzy viscoelastic integro-differential model in this paper. With the aid of some appropriate hypotheses, a unified method and the multiplier technique are implemented to get priori estimates precisely without constructing any auxiliary function. By establishing the estimation of energy function, we derive the stability result of the global solution, and we calculate the estimations of energy attenuation in exponential and polynomial forms, respectively.

\section{Introduction}

In this work, the following fuzzy viscoelastic integro-differential model is considered in a real Hilbert space $X=L^{2}(\Omega)$ :

$$
\left\{\begin{array}{l}
u_{t t}-\Delta u+\int_{0}^{t} g(t-\zeta) \Delta u(\zeta) \mathrm{d} \zeta-|u|^{\gamma} u+\eta^{2} u=0, \quad x \in \Omega, t \in(0, \infty), \\
u(x, t)=0, \quad(x, t) \in \Gamma \times[0, \infty), \\
\left.u(x, t)\right|_{t=0}=u_{0}(x), \\
\left.u_{t}(x, t)\right|_{t=0}=u_{1}(x), \quad x \in \Omega,
\end{array}\right.
$$

where $\Omega$ is an open bounded neighbourhood in $R^{N}$ with $N \geq 3,0<\gamma \leq(2 / N-2)$. Meanwhile, $\Gamma:=\partial \Omega$ is smooth enough. The memory kernel $g(t)$ and the fuzzy number $\eta$ are both positive, and $g(t)$ is locally and absolutely continuous.

As far as the viscoelastic equation is concerned, profound research works have been made in many literature studies [1-7]. For example, the authors in [3] proved a local existence theorem for the next equation:

$$
u_{t t}-\Delta u+\int_{0}^{t} g(t-\zeta) \Delta u(\zeta) \mathrm{d} \zeta=|u|^{\gamma} u,
$$

which is subject to some proper initial data and conditions. In [4], an appropriate Lyapunov-type function was 
introduced by Nasser-eddine Tatar to prove the decay of solutions for the wave equation:

$$
u_{t t}-\Delta u_{t t}-\Delta u+\int_{0}^{t} h(t-\zeta) \Delta u(\zeta) \mathrm{d} \zeta=0
$$

The key contribution of ref. [6] is that the authors demonstrated the decay of the energy function for the next wave equation:

$$
u_{t t}-k_{0} \Delta u+\int_{0}^{t} g(t-\zeta) \operatorname{div}[a(x) \nabla u(\zeta)] \mathrm{d} \zeta+b(x) h\left(u_{t}\right)=0
$$

and some Lyapunov functions were exploited felicitously to deduct more general energy decay results. In [8], a nonlinear hereditary memory evolution equation was considered, and several stability results were given just by means of a simple auxiliary function. The authors in [9] attained analytical and approximate solutions for the cubic Boussinesq equations and modified ones with the aid of the He-Laplace method. Besides, fuzzy synchronization problems have captured the intensive interests of scholars (see, e.g., $[10,11])$, where an adaptive fuzzy backstepping control method was developed in ref. [11] for a sort of uncertain fractional-order nonlinear system.

Generally speaking, in most of the existing works, the presence of auxiliary functions is inevitable, which is exploited to seek the attenuation result of the solution. Accordingly, in the discussion of energy attenuation of solutions for the fuzzy viscoelastic integro-differential model, how to reduce the construction of auxiliary functions has become a problem worth discussing. Taking the integrodifferential abstract equation into account led to fruitful excellent results (see, e.g., [12-14]). In [12], Boussouira et al. proposed a unified method creatively. They derived the decay results for second-order integro-differential equations in the following abstract form:

$$
u^{\prime \prime}+A u-\int_{0}^{t} \beta(t-\zeta) A u(\zeta) \mathrm{d} \zeta=\nabla F(u) .
$$

Also, they put forward an exquisite unified method. With the help of the multiplier method, they accurately described the energy attenuation of the solution of the abstract equation mentioned above.

Inspired by these works, system (1) involved in this paper is an extension of the equation appeared in [12], in which a term with fuzzy coefficient is creatively added. The decay rates in exponential and polynomial forms, respectively, are straightly derived through the unified method. The specific arrangement is made as follows: firstly, in Section 2, several preliminary materials and essential assumptions are listed, and secondly, Section 3 mainly concentrates on the global solution and the estimation of energy attenuation, which are derived by letting $t \longrightarrow \infty$, and the priori estimates are deduced without constructing any auxiliary function. Such outcomes reflect the reliability and effectiveness of the unified method in practice.

\section{Preliminaries}

Throughout this work, the inner product $\langle\cdot, \cdot\rangle$ of $X$ will be utilized in its usual sense, and the norm is defined as follows:

$$
\|u\|=\sqrt{\int_{\Omega}|u(x)|^{2} \mathrm{~d} x}, \quad \forall u \in X .
$$

Note that

$$
u(x, t)=0, \quad(x, t) \in \Gamma \times[0, \infty),
$$

Taking the operator

$$
-\Delta: D(-\Delta) \longrightarrow X,
$$

into consideration, we can verify that

$$
\begin{aligned}
D(-\Delta) & =H^{2}(\Omega) \cap H_{0}^{1}(\Omega) \subset X, \\
D(\nabla) & =H_{0}^{1}(\Omega),
\end{aligned}
$$

where $D(-\Delta)$ is a dense domain. It is evident that, for some positive constant $M$, the linear operator $-\Delta$ is self-adjoint on the real Hilbert space $L^{2}(\Omega)$ and satisfies an inequality similar to the Poincaré inequality [15]:

$$
\langle-\Delta u, u\rangle=\langle\nabla u, \nabla u\rangle=\|\nabla u\|^{2} \geq M\|u\|^{2} .
$$

What is more, we find that $-\Delta$ is accretive due to $\langle-\Delta u, u\rangle \geq 0$.

Now, we give the following assumptions and preliminary materials about the memory kernel $g(t)$.

$\left(H_{1}\right)$ : as far as $g:[0,+\infty) \longrightarrow[0,+\infty)$ is concerned, for some $2<p \leq \infty$ and $k>0$, the function $g$ fulfills the following conditions:

$$
\begin{aligned}
& g(0)>0, \\
& \int_{0}^{\infty} g(\zeta) \mathrm{d} \zeta<1, \\
& g^{\prime} \leq-k g^{(p+1) / p} .
\end{aligned}
$$

Remark 1. If $p=\infty$, then $g^{\prime} \leq-k g$ yields $g(t) \leq C e^{-k t}$ with $t \geq 0$, which indicates that $g(t)$ will decay exponentially.

If $2<p<\infty$, then $g^{\prime} \leq-k g^{(p+1) / p}$ yields

$$
g^{\prime} g^{-1-(1 / p)} \leq-k,
$$

i.e.,

$$
\left(-p g^{-1-(1 / p)+1}\right)^{\prime}=\left(-p g^{-(1 / p)}\right)^{\prime} \leq-k
$$

Next, based on the aforementioned results, the following expression can be obtained by integrating from 0 to $t$ :

$$
-p g^{-(1 / p)}(t)+p g^{-(1 / p)}(0) \leq-k t
$$

namely,

$$
g(t) \leq \frac{1}{\left((k t / p)+g^{-(1 / p)}(0)\right)^{p}} .
$$


This means that $g(t)$ will decay polynomially. Simultaneously, by generalized integral property, if $p \vartheta>1$, it may imply that $g^{9} \in L^{1}(0, \infty)$.

Lemma 1. Suppose that

$$
F(u)=\frac{1}{\gamma+2} \int_{\Omega}|u|^{\gamma+2} \mathrm{~d} x-\frac{\eta^{2}}{2} \int_{\Omega}|u|^{2} \mathrm{~d} x .
$$

The function is Gateaux differentiable for every $u \in D(\nabla), \nabla F(u)=|u|^{\gamma} u-\eta^{2} u$, and

$$
|F(u)| \leq C\|\nabla u\|^{(\gamma / 2)+2} .
$$

Indeed, it is straightforward to see that $F(0)=0, \nabla F(0)=0$. For any pair $u, v \in D(\nabla)$, there exists $c(u)>0$ such that

$$
\begin{aligned}
|\mathrm{DF}(u)(v)| & =|\langle\nabla F(u), v\rangle|=\left|\int_{\Omega} \nabla F(u) v \mathrm{~d} \xi\right|=\left|\int_{\Omega}\left(|u|^{\gamma}-\eta^{2}\right) u v \mathrm{~d} \xi\right| \\
& \leq C\|\nabla u\|^{\gamma+1}\|v\|+\eta^{2} \int_{\Omega}|u v| \mathrm{d} \xi \leq C\|\nabla u\|^{\gamma+1}\|v\|+\eta^{2}\|u\|\|v\| \\
& \leq C\|\nabla u\|^{\gamma+1}\|v\|+\frac{\eta^{2}}{\sqrt{M}}\|\nabla u\|\|v\| \leq c(u)\|v\|,
\end{aligned}
$$

in which $c(u)=C\|\nabla u\|^{\gamma+1}+\left(\eta^{2} / \sqrt{M}\right)\|\nabla u\|$.

For all $u, v \in D(\nabla)$ with $\|\nabla u\|,\|\nabla v\| \leq R$, where $R>0$, it is easily seen that $u-v \in D(\nabla)$. Combining the mean value formula applied in [12] and (10), one can verify that

$$
\begin{aligned}
\|\nabla F(u)-\nabla F(v)\|^{2} & =\left.\int_{\Omega}|| u\right|^{\gamma} u-\eta^{2} u-|v|^{\gamma} v+\left.\eta^{2} v\right|^{2} \mathrm{~d} \xi=\int_{\Omega}\left|\left(|u|^{\gamma} u-|v|^{\gamma} v\right)-\eta^{2}(u-v)\right|^{2} \mathrm{~d} \xi \\
& =\left.\int_{\Omega}|| u\right|^{\gamma} u-\left.|v|^{\gamma} v\right|^{2} \mathrm{~d} \xi+\eta^{2} \int_{\Omega}|u-v|^{2} \mathrm{~d} \xi \\
& \leq C\left(\int_{\Omega}\left(|\nabla u|^{2}+|\nabla v|^{2}\right) \mathrm{d} \xi\right)^{\gamma} \int_{\Omega}|\nabla u-\nabla v|^{2} \mathrm{~d} \xi+\frac{\eta^{2}}{M} \int_{\Omega}|\nabla u-\nabla v|^{2} \mathrm{~d} \xi \\
& \leq C_{R}^{2}\|\nabla u-\nabla v\|^{2} .
\end{aligned}
$$

That is, some positive constant $C_{R}=\left[C\left(\int_{\Omega}\left(|\nabla u|^{2}+\right.\right.\right.$ $\left.\left.\left.|\nabla v|^{2}\right) \mathrm{d} \xi\right)^{\gamma}+\left(\eta^{2} / M\right)\right]^{(1 / 2)}$ can be found to satisfy

$$
\|\nabla F(u)-\nabla F(v)\| \leq C_{R}\|\nabla u-\nabla v\| .
$$

By letting $\psi(u)=u^{(\gamma / 2)}$, it is easy to see that $\psi:[0, \infty) \longrightarrow[0, \infty)$ is continuous and strictly increasing. Now, we suppose that $|\langle\nabla F(u), u\rangle| \leq C\|\nabla u\|^{(\gamma / 2)+2}$, that is,

$$
\begin{aligned}
\langle\nabla F(u), u\rangle & =\left\langle\left(|u|^{\gamma}-\eta^{2}\right) u, u\right\rangle=\int_{\Omega}\left(|u(\xi)|^{\gamma}-\eta^{2}\right) u(\xi)^{2} \mathrm{~d} \xi \\
& \leq C\|\nabla u\|^{(\gamma / 2)}\|\nabla u\|^{2}=C\|\nabla u\|^{(\gamma / 2)+2}, \quad \forall u \in D(\nabla) .
\end{aligned}
$$

For every $u \in D(\nabla)$,

$$
\begin{aligned}
|F(u)| & \leq \int_{0}^{1}|\langle\nabla F(t u), u\rangle| \mathrm{d} t \leq\|\nabla u(t)\|^{2} \int_{0}^{1}(t\|\nabla u(t)\|)^{(\gamma / 2)} t \mathrm{~d} t \\
& \leq C\|\nabla u(t)\|^{2+(\gamma / 2)},
\end{aligned}
$$

which yields

$$
\left.\left|\frac{1}{\gamma+2} \int_{\Omega}\right| u\right|^{\gamma+2} \mathrm{~d} x-\frac{\eta^{2}}{2} \int_{\Omega}|u|^{2} \mathrm{~d} x \mid \leq\|\nabla u(t)\|^{2+(\gamma / 2)} .
$$

Remark 2. For any $0<T \leq \infty$, by taking the measurable function $u:(0, T) \longrightarrow X$ into consideration, it is known that both $\|u\|_{1}=\int_{0}^{t}\|u(t)\| \mathrm{d} t$ and $\|u\|_{\infty}=\operatorname{esssup}_{t \in[0, T]}\|u(t)\|$ are finite.

For any $f \in L^{1}(0, T)$ and $u \in L^{1}(0, T ; X)$, we denote the convolution as follows:

$$
f^{*} u(t)=\int_{0}^{t} f(t-s) u(s) \mathrm{d} s, \quad 0 \leq t \leq T .
$$

Aiming to facilitate the subsequent narration, we proceed to present the next useful lemmas.

Lemma 2. Consider a nonnegative nonincreasing function $\mathscr{E}(t)$ with $0 \leq t<\infty$. If there exists a negative constant $T$ such that

$$
\int_{t}^{\infty} \mathscr{E}(s) \mathrm{d} s \leq T \mathscr{E}(t)
$$

then 


$$
\mathscr{E}(t) \leq \mathscr{E}(0) e^{1-(t / T)}, \quad \forall t \geq T
$$

Proof. Let

$$
f(x)=e^{(x / T)} \int_{x}^{\infty} \mathscr{E}(s) \mathrm{d} s, \quad \forall x \geq 0 .
$$

Then its derivative is calculated as

$$
f^{\prime}(x)=\frac{e^{(x / T)}}{T}\left(\int_{x}^{\infty} \mathscr{E}(s) \mathrm{d} s-T \mathscr{E}(x)\right) .
$$

Considering that $\int_{t}^{\infty} \mathscr{E}(s) \mathrm{d} s \leq T \mathscr{E}(t)$, we have

$$
f(x) \leq f(0)=\int_{0}^{\infty} \mathscr{E}(s) \mathrm{d} s \leq T \mathscr{E}(0) .
$$

This implies that

$$
\int_{x}^{\infty} \mathscr{E}(s) \mathrm{d} s \leq T \mathscr{E}(0) e^{-(x / T)} .
$$

On the other hand, since $\mathscr{E}$ is nonnegative and nonincreasing,

$$
\int_{x}^{\infty} \mathscr{E}(s) \mathrm{d} s \geq \int_{x}^{x+T} 1 \cdot \mathscr{E}(s) \mathrm{d} s \geq T \mathscr{E}(x+T) .
$$

Combining (30) with (31), we get

$$
\mathscr{E}(x+T) \leq \mathscr{E}(0) e^{-(x / T)} .
$$

Taking $t=x+T$, by $x \geq 0$, formula (26) is obtained naturally.

Lemma 3. Let $\mathscr{E}(t)$ be a nonnegative and nonincreasing function on $[0, \infty)$. If

$$
\int_{T}^{\infty} \mathscr{E}^{1+m}(t) \mathrm{d} t \leq C \mathscr{E}^{m}(0) \mathscr{E}(T), \quad \forall T \geq T_{0}
$$

where $m, C$, and $T_{0}$ are all positive constants. Then, for arbitrary $t \in[0, \infty)$, it holds that

$$
\mathscr{E}(t) \leq \mathscr{E}(0)\left(\frac{\left(C+T_{0}\right)(1+m)}{m t+C+T_{0}}\right)^{(1 / m)} .
$$

The proof of Lemma 3 is analogous to that of Lemma 2, and hence, it is omitted here.

Let $u_{i} \in X(i=0,1)$. Now, let us discuss the problem as follows:

$$
\left\{\begin{array}{l}
u_{t t}-\Delta u+\int_{0}^{t} g(t-\zeta) \Delta u(\zeta) \mathrm{d} \zeta-|u|^{\gamma} u+\eta^{2} u=0, \quad 0<t<\infty \\
u(0)=u_{0}, \\
\left.u\right|_{t}(0)=u_{1} .
\end{array}\right.
$$

For any $0 \leq t \leq T,(T>0)$, with the aid of the description in [12], a mild solution of (35) can be described as follows:

$$
\begin{aligned}
u(t)= & u_{0} S(t)+\int_{0}^{t} S(\zeta) u_{1} \mathrm{~d} \zeta+\int_{0}^{t} 1 \\
& * S(t-\zeta)\left(|u(\zeta)|^{\gamma}-\eta^{2}\right) u(\zeta) \mathrm{d} \zeta
\end{aligned}
$$

where

$$
1^{*} S(t-\zeta)=\int_{0}^{t-\zeta} S(\tau) \mathrm{d} \tau, \quad \zeta \leq t \leq T+\zeta,
$$

and $\{S(t)\}$ is the resolvent for the corresponding linear problem of (35).

As far as the weak solution is concerned, $u$ is a function in $C^{1}([0, T] ; X) \cap C([0, T] ; D(\nabla))$ and satisfies

$$
\begin{aligned}
\frac{\mathrm{d}}{\mathrm{d} t} & \left\langle u_{t}(t), v\right\rangle+\langle\nabla u, \nabla v\rangle-\left\langle\int_{0}^{t} g(t-\zeta) \nabla u(\zeta) \mathrm{d} \zeta, \nabla v\right\rangle \\
& =\left\langle\left(|u|^{\gamma}-\eta^{2}\right) u, v\right\rangle,
\end{aligned}
$$

$\forall v \in D(\nabla),\left\langle u_{t}(t), v\right\rangle \in C^{1}([0, T])$ and $0 \leq t \leq T$.

Local existence, uniqueness, and regularity for (1) are naturally guaranteed by the result in [12].

Considering a mild solution $u$ of $(1)(t \in[0, T])$, and using $u_{t}$ as a multiplier, the multiplier method can be used to get the energy of $u$ as follows:

$$
\begin{aligned}
\mathscr{E}_{u}(t)= & \frac{1}{2}\left(\left\|u_{t}\right\|^{2}+\left(\left(1-\int_{0}^{t} g(\zeta) \mathrm{d} \zeta\right)\|\nabla u\|^{2}\right.\right. \\
& \left.+\int_{0}^{t} g(t-\zeta)\|\nabla u(\zeta)-\nabla u(t)\|^{2} \mathrm{~d} \zeta\right) \\
& -\frac{1}{\gamma+2} \int_{\Omega}|u|^{\gamma+2} \mathrm{~d} x+\frac{\eta^{2}}{2} \int_{\Omega}|u|^{2} \mathrm{~d} x
\end{aligned}
$$

Next, it is necessary to discuss the decay of $\mathscr{E}_{u}(t)$.

Consider that $u$ is a strong solution of problem (1) on an interval $[0, T]$. By taking derivative of (39), we obtain 


$$
\begin{aligned}
\frac{\mathrm{d}}{\mathrm{d} t} \mathscr{E}_{u}(t)= & \left\langle u_{t t}, u_{t}\right\rangle+\left\langle\nabla u, \nabla u_{t}\right\rangle-\frac{g(t)}{2}\|\nabla u\|^{2}-\frac{1}{2}\left(\int_{0}^{t} g(\zeta) \mathrm{d} \zeta\right) \frac{\mathrm{d}}{\mathrm{d} t}\|\nabla u\|^{2} \\
& +\frac{1}{2} \int_{0}^{t} g^{\prime}(t-\zeta)\|\nabla u(t)-\nabla u(\zeta)\|^{2} \mathrm{~d} \zeta-\int_{0}^{t} g(t-\zeta)\left\langle\nabla u(\zeta)-\nabla u(t), \nabla u_{t}(t)\right\rangle \mathrm{d} \zeta-\left\langle\left(|u|^{\gamma}-\eta^{2}\right) u, u_{t}\right\rangle \\
= & \left\langle u_{t t}, u_{t}\right\rangle+\left\langle\nabla u, \nabla u_{t}\right\rangle-\frac{g(t)}{2}\|\nabla u\|^{2}-\frac{1}{2}\left(\int_{0}^{t} g(\zeta) \mathrm{d} \zeta\right) \frac{\mathrm{d}}{\mathrm{d} t}\|\nabla u\|^{2}+\frac{1}{2} \int_{0}^{t} g^{\prime}(t-\zeta)\|\nabla u(t)-\nabla u(\zeta)\|^{2} \mathrm{~d} \zeta \\
& -\left\langle\int_{0}^{t} g(t-\zeta) \nabla u(\zeta) \mathrm{d} \zeta, \nabla u_{t}\right\rangle+\frac{1}{2}\left(\int_{0}^{t} g(\zeta) \mathrm{d} \zeta\right) \frac{\mathrm{d}}{\mathrm{d} t}\|\nabla u\|^{2}-\left\langle\left(|u|^{\gamma}-\eta^{2}\right) u, u_{t}\right\rangle \\
= & \left\langle u_{t t}, u_{t}\right\rangle+\left\langle-\Delta u, u_{t}\right\rangle+\left\langle\int_{0}^{t} g(t-\zeta) \Delta u(\zeta) \mathrm{d} \zeta, u_{t}\right\rangle-\left\langle\left(|u|^{\gamma}-\eta^{2}\right) u, u_{t}\right\rangle-\frac{g(t)}{2}\|\nabla u\|^{2} \\
& +\frac{1}{2} \int_{0}^{t} g^{\prime}(t-\zeta)\|\nabla u(t)-\nabla u(\zeta)\|^{2} \mathrm{~d} \zeta \\
= & -\frac{g(t)}{2}\|\nabla u\|^{2}+\frac{1}{2} \int_{0}^{t} g^{\prime}(t-\zeta)\|\nabla u(t)-\nabla u(\zeta)\|^{2} \mathrm{~d} \zeta .
\end{aligned}
$$

In view of the facts that $g \leq 0$ and $g^{\prime} \geq 0$, it follows from these assumptions that

$$
\begin{aligned}
\frac{\mathrm{d}}{\mathrm{d} t} \mathscr{C}_{u}(t)= & -\frac{g(t)}{2}\|\nabla u\|^{2} \\
& +\frac{1}{2} \int_{0}^{t} g^{\prime}(t-\zeta)\|\nabla u(t)-\nabla u(\zeta)\|^{2} \mathrm{~d} \zeta \leq 0,
\end{aligned}
$$

that is, $\mathscr{E}_{u}(t)$ is decreasing. One can draw a similar conclusion for mild solutions. In a word, if the initial conditions are small sufficiently, the solution of model (1) exists globally.

Theorem 1. Assume that $H_{1}$ holds. For any $u_{0} \in D(\nabla)$ and $u_{1} \in X$, if there is a positive scalar $\rho_{0}$ such that

$$
\left\|\nabla u_{0}\right\|+\left\|u_{1}\right\|<\rho_{0},
$$

then there is a unique mild solution u for problem (1). Besides, for arbitrary $t \in[0, \infty)$,

$$
\begin{aligned}
& \mathscr{E}_{u}(t)>0, \\
& \mathscr{E}_{u}(t) \leq \mathscr{E}_{u}(0) \leq \rho_{0}^{2},
\end{aligned}
$$

$$
\begin{aligned}
\mathscr{E}_{u}(t) & \geq \frac{1}{2}\left\|u_{t}\right\|^{2}+\frac{1-\int_{0}^{\infty} g(\zeta) \mathrm{d} \zeta}{4}\|\nabla u(t)\|^{2}, \\
\|\nabla u(t)\|^{2+(\gamma / 2)} & \leq \frac{1-\int_{0}^{\infty} g(\zeta) \mathrm{d} \zeta}{4} .
\end{aligned}
$$

Furthermore, $u$ is a strong solution of (1), provided that $u_{0} \in D(-\Delta)$ and $u_{1} \in D(\nabla)$.

Proof. Assume that a maximal definition interval for the mild solution of problem (1) is $[0, T)$, and $\ell=\left(1-\int_{0}^{\infty} g(\zeta) \mathrm{d} \zeta\right) / 2$. According to Lemma 1 , one gets $(\mid(1 / \gamma+2)) \int_{\Omega}\left|u_{0}\right|^{\gamma+2} \mathrm{~d} x \mid \leq\left\|\nabla u_{0}\right\|^{2+(\gamma / 2)}$. Besides, equation (39) implies

$$
\begin{aligned}
\mathscr{E}_{u}(0)= & \frac{1}{2}\left(\left\|u_{1}\right\|^{2}+\left\|\nabla u_{0}\right\|^{2}\right)-\frac{1}{\gamma+2} \int_{\Omega}\left|u_{0}\right|^{\gamma+2} \mathrm{~d} x \\
& +\frac{\eta^{2}}{2} \int_{\Omega}\left|u_{0}\right|^{2} \mathrm{~d} x .
\end{aligned}
$$

If $\left\|\nabla u_{0}\right\|^{(\gamma / 2)}<(\ell / 2)$, we get

$$
\begin{aligned}
\left.\left|\frac{1}{\gamma+2} \int_{\Omega}\right| u_{0}\right|^{\gamma+2} \mathrm{~d} x-\frac{\eta^{2}}{2} \int_{\Omega}\left|u_{0}\right|^{2} \mathrm{~d} x \mid & \leq\left.\left|\frac{1}{\gamma+2} \int_{\Omega}\right| u_{0}\right|^{\gamma+2} \mathrm{~d} x\left|+\frac{\eta^{2}}{2}\right| \int_{\Omega}\left|u_{0}\right|^{2} \mathrm{~d} x \mid \\
& \leq\left\|\nabla u_{0}\right\|^{2+(\gamma / 2)}+\frac{\eta^{2}}{2 M}\left\|\nabla u_{0}\right\|^{2}<\frac{\ell}{2}\left\|\nabla u_{0}\right\|^{2}+\frac{\eta^{2}}{2 M}\left\|\nabla u_{0}\right\|^{2} \leq \frac{\tilde{\ell}}{2}\left\|\nabla u_{0}\right\|^{2},
\end{aligned}
$$

where $\tilde{\ell} \geq \ell+\left(\eta^{2} / M\right)$.

Thus, it is naturally acquired that

$$
-\frac{1}{\gamma+2} \int_{\Omega}\left|u_{0}\right|^{\gamma+2} \mathrm{~d} x+\frac{\eta^{2}}{2} \int_{\Omega}\left|u_{0}\right|^{2} \mathrm{~d} x \geq-\frac{\tilde{\ell}}{2}\left\|\nabla u_{0}\right\|^{2}
$$

Consequently, $\mathscr{E}_{u}(0) \geq(1 / 2)\left(\left\|u_{1}\right\|^{2}+(1-\tilde{\ell})\left\|\nabla u_{0}\right\|^{2}\right) \geq 0$. Put $\rho_{0}=(\tilde{\ell} / 2)^{(1 / 2)+(2 / \gamma)}$. Suppose that $u_{0} \in D(\nabla)$ and $u_{1} \in X$ satisfy

$$
\left\|\nabla u_{0}\right\|+\left\|u_{1}\right\|<\rho_{0}
$$


Utilizing the amplification method, i.e.,

$$
\begin{aligned}
\mathscr{E}_{u}(0) & \leq \frac{1}{2}\left(\left\|u_{1}\right\|^{2}+\left\|\nabla u_{0}\right\|^{2}\right) \leq\left(\left\|u_{1}\right\|^{2}+\left\|\nabla u_{0}\right\|^{2}\right) \\
& \leq\left(\left(\left\|u_{1}\right\|+\left\|\nabla u_{0}\right\|\right)\right)^{2} \leq \rho_{0}^{2}=\left(\frac{\tilde{\ell}}{2}\right)^{1+(4 / \gamma)},
\end{aligned}
$$

we derive that $\left(2 \mathscr{E}_{u}(0) / \widetilde{\ell}\right)^{(\gamma / 4)}<(\tilde{\ell} / 2)$ and

$$
\begin{aligned}
\mathscr{E}_{u}(t) \geq & \frac{1}{2}\left\|u_{t}\right\|^{2}+\frac{\tilde{\ell}}{2}\|\nabla u(t)\|^{2} \geq \frac{1}{2}\left\|u_{t}\right\|^{2} \\
& +\left[\frac{1-\int_{0}^{\infty} g(\zeta) \mathrm{d} \zeta}{4}+\frac{\eta^{2}}{2 M}\right]\|\nabla u(t)\|^{2} \\
\geq & \frac{1}{2}\left\|u_{t}\right\|^{2}+\frac{1-\int_{0}^{\infty} g(\zeta) \mathrm{d} \zeta}{4}\|\nabla u(t)\|^{2}, \quad \forall t \in[0, T) .
\end{aligned}
$$

So, the energy $\mathscr{E}_{u}$ is well bounded and the solution $u$ exists globally. The proof of the aforementioned formula is based on the idea of reductio ad absurdum, and the detailed process is omitted here.

\section{Main Results}

In this sequel, without invoking any auxiliary function, we put forward the main result as follows.

Theorem 2. Assume that $\left(H_{1}\right)$ holds. Given $S \geq S_{0}>0$. For each pair $\left(u_{0}, u_{1}\right) \in D(\nabla) \times X$, if $\left\|u_{1}\right\|+\left\|\nabla u_{0}\right\|<\rho_{0}$ with $\rho_{0}$ being a positive constant, then there is some positive constant $C$ ensuring that the mild solution of model (1) satisfies the next property:

$$
\int_{S}^{\infty} \mathscr{E}_{u}^{1+(1 / p)}(t) \mathrm{d} t \leq C \mathscr{E}_{u}^{(1 / p)}(0) \mathscr{E}_{u}(S)
$$

Specifically,

$$
\begin{aligned}
& \mathscr{E}_{u}(t) \leq \mathscr{E}_{u}(0) e^{1-C t}, \quad p=\infty, \\
& \mathscr{E}_{u}(t) \leq \mathscr{E}_{u}(0)\left(\frac{p+1}{p+C t}\right)^{p}, \quad 2<p<\infty .
\end{aligned}
$$

Proof. By Theorem 1, we know that the solution of (1) is global. Moreover, it is easy to check that the solution is strong if $u_{0} \in D(-\Delta)$ and $u_{1} \in D(\nabla)$. Aiming to show (53), we begin to focus on the formula as follows

$$
\begin{aligned}
\int_{S}^{T} \mathscr{E}_{u}^{1+(1 / p)}(t) \mathrm{d} t= & \frac{1}{2} \int_{S}^{T} \mathscr{E}_{u}^{(1 / p)}(t)\left\|u_{t}\right\|^{2} \mathrm{~d} t+\frac{1}{2} \int_{S}^{T} \mathscr{E}_{u}^{(1 / p)}(t)\left(1-\int_{0}^{t} g(\zeta) \mathrm{d} \zeta\right)\|\nabla u\|^{2} \mathrm{~d} t \\
& +\frac{1}{2} \int_{S}^{T} \mathscr{E}_{u}^{(1 / p)}(t) \int_{0}^{t} g(t-\zeta)\|\nabla u(\zeta)-\nabla u(t)\|^{2} \mathrm{~d} \zeta \mathrm{d} t \\
& -\frac{1}{\gamma+2} \int_{S}^{T} \mathscr{E}_{u}^{(1 / p)}(t) \int_{\Omega}|u|^{\gamma+2} \mathrm{~d} x \mathrm{~d} t+\frac{\eta^{2}}{2} \int_{S}^{T} \mathscr{E}_{u}^{(1 / p)}(t) \int_{\Omega}|u|^{2} \mathrm{~d} x \mathrm{~d} t .
\end{aligned}
$$

Next, our task is introducing an approach for controlling every term of the right hand of equation (55) via multiplier methods. At the beginning, we propose the following lemma.
Lemma 4. Suppose that $\varphi(t): R_{+} \longrightarrow R_{+}$is a multiplier, fulfilling that $\varphi^{\prime}(t)<0$. Then for any positive constant $T$ with $T \geq S \geq S_{0}$, there exists $C>0$ such that

$$
\begin{aligned}
& \frac{1}{2} \int_{S}^{T} \varphi(t)\left\|u_{t}\right\|^{2} \mathrm{~d} t+\frac{1}{2} \int_{S}^{T} \varphi(t)\left(1-\int_{0}^{t} g(\zeta) \mathrm{d} \zeta\right)\|\nabla u(t)\|^{2} \mathrm{~d} t \\
& -\frac{1}{\gamma+2} \int_{S}^{T} \varphi(t) \int_{\Omega}|u|^{\gamma+2} \mathrm{~d} x \mathrm{~d} t+\frac{\eta^{2}}{2} \int_{S}^{T} \varphi(t) \int_{\Omega}|u|^{2} \mathrm{~d} x \mathrm{~d} t \leq C \varphi(0) \mathscr{E}_{u}(S) .
\end{aligned}
$$

Proof. Firstly, an inner product of model (1) with the multiplication of $u$ and $\varphi(t)$ should be taken. Next, integrating it on the closed interval $[S, T]$, the following description is now obtained:

$$
\int_{S}^{T} \varphi(t)\left\langle u_{t t}-\Delta u+\int_{0}^{t} g(t-\zeta) \Delta u(\zeta) \mathrm{d} \zeta, u(t)\right\rangle \mathrm{d} t=\int_{S}^{T} \varphi(t)\left\langle\left(|u|^{\gamma}-\eta^{2}\right) u(t), u(t)\right\rangle \mathrm{d} t
$$


Integrating by parts, we get

$$
\begin{aligned}
& \int_{S}^{T} \varphi(t)\left\langle u_{t t}, u(t)\right\rangle \mathrm{d} t=\int_{S}^{T} \varphi(t) \frac{\mathrm{d}}{\mathrm{d} t}\left\langle u_{t}, u(t)\right\rangle \mathrm{d} t-\int_{S}^{T} \varphi(t) \frac{\mathrm{d}}{\mathrm{d} t}\left\langle u_{t}, u_{t}\right\rangle \mathrm{d} t \\
& \quad=\left[\varphi(t)\left\langle u_{t}, u(t)\right\rangle\right]_{S}^{T}-\int_{S}^{T} \varphi^{\prime}(t) \frac{\mathrm{d}}{\mathrm{d} t}\left\langle u_{t}, u(t)\right\rangle \mathrm{d} t-\int_{S}^{T} \varphi(t) \frac{\mathrm{d}}{\mathrm{d} t}\left\|u_{t}\right\|^{2} \mathrm{~d} t \\
& \int_{S}^{T} \varphi(t)\langle-\Delta u, u(t)\rangle \mathrm{d} t=\int_{S}^{T} \varphi(t)\langle\nabla u, \nabla u\rangle \mathrm{d} t=\int_{S}^{T} \varphi(t)\|\nabla u\|^{2} \mathrm{~d} t, \\
& \int_{S}^{T} \varphi(t)\left\langle\int_{0}^{t} g(t-\zeta) \Delta u(\zeta) \mathrm{d} \zeta, u(t)\right\rangle \mathrm{d} t \\
& =-\int_{S}^{T} \varphi(t)\left\langle\int_{0}^{t} g(t-\zeta) \nabla u(\zeta) \mathrm{d} \zeta, \nabla u(t)\right\rangle \mathrm{d} t \\
& =-\int_{S}^{T} \varphi(t)\left\langle\int_{0}^{t} g(t-\zeta)(\nabla u(\zeta)-\nabla u(t)) \mathrm{d} \zeta, \nabla u(t)\right\rangle \mathrm{d} t, \\
& \left.-\int_{S}^{T} \varphi(t) \int_{0}^{t} g(t-\zeta)\langle\nabla u(t)), \nabla u(t)\right\rangle \mathrm{d} \zeta \mathrm{d} t \\
& \quad=-\int_{S}^{T} \varphi(t)\left\langle\int_{0}^{t} g(t-\zeta)(\nabla u(\zeta)-\nabla u(t)) \mathrm{d} \zeta, \nabla u(t)\right\rangle \mathrm{d} t \\
& \quad-\int_{S}^{T} \varphi(t)\left(\int_{0}^{t} g(\zeta) \mathrm{d} \zeta\right)\|\nabla u\|^{2} \mathrm{~d} t, \\
& \quad+\int_{S}^{T} \varphi \varphi^{\prime}(t)\left\langle u_{t}, u(t)\right\rangle \mathrm{d} t+\int_{S}^{T} \varphi(t)\left\langle\left(|u|^{\gamma}-\eta^{2}\right) u(t), u(t)\right\rangle \mathrm{d} t-\left[\varphi(t)\left\langle u_{t}(t), u(t)\right\rangle\right]_{S}^{T} \\
& \int_{S}^{T} \varphi(t)\left(1-\int_{0}^{t} g(\zeta) \mathrm{d} \zeta\right)\|\nabla u(t)\|^{2} \mathrm{~d} t \\
& =\int_{S}^{T} \varphi(t)\left\|u_{t}\right\|^{2} \mathrm{~d} t+\int_{S}^{T} \varphi(t)\left\langle\int_{0}^{t} g(t-\zeta)(\nabla u(\zeta)-\nabla u(t)) \mathrm{d} \zeta, \nabla u(t)\right\rangle \mathrm{d} t \\
&
\end{aligned}
$$

Applying Schwartz inequality $\forall \varepsilon_{1}>0$, we have

$$
\begin{aligned}
& \int_{S}^{T} \varphi(t)\|\nabla u(t)\| \int_{0}^{t} g(t-\zeta)\|\nabla u(\zeta)-\nabla u(t)\| \mathrm{d} \zeta \mathrm{d} t \\
& \quad=\int_{S}^{T} \sqrt{\varphi(t)} \sqrt{\varphi(t)}\|\nabla u(t)\| \int_{0}^{t} g(t-\zeta)\|\nabla u(\zeta)-\nabla u(t)\| \mathrm{d} \zeta \mathrm{d} t \\
& \quad \leq \frac{\varepsilon_{1}}{2} \int_{S}^{T} \varphi(t)\|\nabla u(t)\|^{2} \mathrm{~d} t+\frac{1}{2 \varepsilon_{1}} \int_{S}^{T} \varphi(t)\left(\int_{0}^{t} g(t-\zeta)\|\nabla u(\zeta)-\nabla u(t)\| \mathrm{d} \zeta\right)^{2} \mathrm{~d} t .
\end{aligned}
$$

Taking the integrability of $g^{9}$ and the assumption that $g^{\prime} \leq-k g^{(p+1) / p}$ into consideration, with the help of Hölder inequality and the description of $\mathscr{E}_{u}^{\prime}(t)$, we have 


$$
\begin{aligned}
& \int_{S}^{T} \varphi(t)\left(\int_{0}^{t} g(t-\zeta)\|\nabla u(\zeta)-\nabla u(t)\| \mathrm{d} \zeta\right)^{2} \mathrm{~d} t \\
& \quad=\int_{S}^{T} \varphi(t)\left(\int_{0}^{t} g^{(p-1) / 2 p}(t-\zeta) g^{(p+1) / 2 p}(t-\zeta)\|\nabla u(\zeta)-\nabla u(t)\| \mathrm{d} \zeta\right)^{2} \mathrm{~d} t \\
& \quad \leq \int_{S}^{T} \varphi(t)\left(\int_{0}^{t} g^{(p-1) / p}(\zeta) \mathrm{d} \zeta\right)\left(\int_{0}^{t} g^{(p+1) / p}(t-\zeta)\|\nabla u(\zeta)-\nabla u(t)\|^{2} \mathrm{~d} \zeta\right) \mathrm{d} t \\
& \quad \leq\left(\int_{0}^{\infty} g^{(p-1) / p}(\zeta) \mathrm{d} \zeta\right) \int_{S}^{T} \varphi(t)\left(\int_{0}^{t} g^{(p+1) / p}(t-\zeta)\|\nabla u(\zeta)-\nabla u(t)\|^{2} \mathrm{~d} \zeta\right) \mathrm{d} t \\
& \quad \leq-\frac{1}{k}\left(\int_{0}^{\infty} g^{(p-1) / p}(\zeta) \mathrm{d} \zeta\right) \int_{S}^{T} \varphi(t)\left(\int_{0}^{t} g^{\prime}(t-\zeta)\|\nabla u(\zeta)-\nabla u(t)\|^{2} \mathrm{~d} \zeta\right) \mathrm{d} t \\
& \quad \leq-\frac{2}{k}\left(\int_{0}^{\infty} g^{(p-1) / p}(\zeta) \mathrm{d} \zeta\right) \int_{S}^{T} \varphi(0) \mathscr{E}_{u}^{\prime}(t) \mathrm{d} t \\
& \quad=-\frac{2}{k}\left(\int_{0}^{\infty} g^{(p-1) / p}(\zeta) \mathrm{d} \zeta\right) \varphi(0)\left(\mathscr{E}_{u}(T)-\mathscr{E}_{u}(S)\right) \\
& \quad \leq \frac{2}{k} \varphi(0) \mathscr{E}_{u}(S)\left(\int_{0}^{\infty} g^{(p-1) / p}(\zeta) \mathrm{d} \zeta\right) .
\end{aligned}
$$

Combining (62) and (63), we get

$$
\begin{aligned}
& \int_{S}^{T} \varphi(t)\left|\left\langle\int_{0}^{t} g(t-\zeta)(\nabla u(\zeta)-\nabla u(t)) \mathrm{d} \zeta, \nabla u(t)\right\rangle\right| \mathrm{d} t \\
& \quad=\int_{S}^{T} \varphi(t)\|\nabla u(t)\| \int_{0}^{t} g(t-\zeta)\|\nabla u(\zeta)-\nabla u(t)\| \mathrm{d} \zeta \mathrm{d} t \\
& \quad \leq \frac{\varepsilon_{1}}{2} \int_{S}^{T} \varphi(t)\|\nabla u(t)\|^{2} \mathrm{~d} t+\frac{1}{k \varepsilon_{1}} \varphi(0) \mathscr{E}_{u}(S)\left(\int_{0}^{\infty} g^{(p-1) / p}(\zeta) \mathrm{d} \zeta\right) .
\end{aligned}
$$

In view of (45), we have

$$
\begin{aligned}
\frac{1}{2}\|u(t)\|^{2} & \leq \mathscr{E}_{u}(t), \\
\frac{1}{2}\|\nabla u(t)\|^{2} & \leq \frac{2 \mathscr{E}_{u}(t)}{1-\int_{0}^{\infty} g(\zeta) \mathrm{d} \zeta} .
\end{aligned}
$$

Thus, we obtain

$$
\frac{1}{2}\|u(t)\|^{2} \leq \frac{1}{M}\left(\frac{1}{2}\|\nabla u(t)\|^{2}\right) \leq \frac{(2 / M) \mathscr{E}_{u}(t)}{1-\int_{0}^{\infty} g(\zeta) \mathrm{d} \zeta}
$$

Therefore, the following result is arrived:

$$
\begin{aligned}
& \begin{aligned}
\left|\left\langle u_{t}(t), u(t)\right\rangle\right| & =\left|\int_{0}^{T} u_{t}(t) u(t) \mathrm{d} t\right| \leq \frac{1}{2} \int_{0}^{T}\left|u_{t}(t)\right|^{2} \mathrm{~d} t+\frac{1}{2} \int_{0}^{T}|u(t)|^{2} \mathrm{~d} t \\
\leq \frac{1}{2}\|u(t)\|^{2}+\frac{(2 / M) \mathscr{E}_{u}(t)}{1-\int_{0}^{\infty} g(\zeta) \mathrm{d} \zeta} \leq\left(1+\frac{(2 / M)}{1-\int_{0}^{\infty} g(\zeta) \mathrm{d} \zeta}\right) \mathscr{E}_{u}(t) . & \square-\int_{S}^{T} \varphi_{t}(t) d t=\varphi(S)-\varphi(T) \leq \varphi(S) \leq \varphi(0) .
\end{aligned} \\
& (t) \leq \mathscr{E}_{u}(S) \text {, we get }
\end{aligned}
$$

By $\varphi_{t}(t)<0$ and $\mathscr{E}_{u}(t) \leq \mathscr{E}_{u}(S)$, we get 
Therefore,

$$
\begin{aligned}
\int_{S}^{T} \varphi_{t}(t)\left\langle u_{t}(t), u(t)\right\rangle \mathrm{d} t & \leq-\int_{S}^{T} \varphi_{t}(t)\left\langle u_{t}(t), u(t)\right\rangle \mathrm{d} t \\
& \leq-\left(1+\frac{(2 / M)}{1-\int_{0}^{\infty} g(\zeta) \mathrm{d} \zeta}\right) \int_{S}^{T} \varphi_{t}(t) \mathscr{E}_{u}(t) \mathrm{d} t \\
& \leq \mathscr{E}_{u}(S)\left(1+\frac{(2 / M)}{1-\int_{0}^{\infty} g(\zeta) \mathrm{d} \zeta}\right)\left(-\int_{S}^{T} \varphi_{t}(t) \mathrm{d} t\right) \\
& \leq \mathscr{E}_{u}(S) \varphi(0)\left(1+\frac{(2 / M)}{1-\int_{0}^{\infty} g(\zeta) \mathrm{d} \zeta}\right)
\end{aligned}
$$

By the condition imposed on the proof of Theorem 1,

$$
\|\nabla u(t)\|^{(\gamma / 2)} \leq \frac{1-\int_{0}^{\infty} g(\zeta) \mathrm{d} \zeta}{4}
$$

one has

$$
\begin{aligned}
& \int_{S}^{T} \varphi(t)\left\langle\left(|u|^{\gamma}-\eta^{2}\right) u(t), u(t)\right\rangle \mathrm{d} t \leq \int_{S}^{T} \varphi(t)\|\nabla u(t)\|^{2+(\gamma / 2)} \mathrm{d} t \\
& \quad \leq \frac{1}{4} \int_{S}^{T} \varphi(t)\left(1-\int_{0}^{t} g(\zeta) \mathrm{d} \zeta\right)\|\nabla u(t)\|^{2} \mathrm{~d} t .
\end{aligned}
$$

Considering that both $\varphi(t)$ and $\mathscr{E}(t)$ are decreasing, from (67), we deduce

$$
-\left[\varphi(t)\left\langle u_{t}(t), u(t)\right\rangle\right]_{S}^{T} \leq 2 \varphi(0)\left(1+\frac{(2 / M)}{1-\int_{0}^{\infty} g(\zeta) \mathrm{d} \zeta}\right) \mathscr{E}_{u}(S) .
$$

Based on equation (61), it is trivially shown that

$$
\begin{aligned}
& \int_{S}^{T} \varphi(t)\left(1-\int_{0}^{t} g(\zeta) \mathrm{d} \zeta\right)\|\nabla u(t)\|^{2} \mathrm{~d} t \\
& \quad=\int_{S}^{T} \varphi(t)\left\|u_{t}\right\|^{2} \mathrm{~d} t+\int_{S}^{T} \varphi(t)\left\langle\int_{0}^{t} g(t-\zeta)(\nabla u(\zeta)-\nabla u(t)) \mathrm{d} \zeta, \nabla u(t)\right\rangle \mathrm{d} t \\
& \quad+\int_{S}^{T} \varphi^{\prime}(t)\left\langle u_{t}, u(t)\right\rangle \mathrm{d} t+\int_{S}^{T} \varphi(t)\left\langle\left(|u|^{\gamma}-\eta^{2}\right) u(t), u(t)\right\rangle \mathrm{d} t-\left[\varphi(t)\left\langle u_{t}(t), u(t)\right\rangle\right]_{S}^{T} \\
& \leq \int_{S}^{T} \varphi(t)\left\|u_{t}\right\|^{2} \mathrm{~d} t+\frac{\varepsilon_{1}}{2} \int_{S}^{T} \varphi(t)\|\nabla u(t)\|^{2} \mathrm{~d} t+\frac{1}{k \varepsilon_{1}} \varphi(0) \mathscr{E}_{u}(S)\left(\int_{0}^{\infty} g(p-1 / p)(\zeta) \mathrm{d} \zeta\right) \\
& \quad+\mathscr{E}_{u}(S) \varphi(0)\left(1+\frac{(2 / M)}{1-\int_{0}^{\infty} g(\zeta) \mathrm{d} \zeta}\right)+\frac{1}{4} \int_{S}^{T} \varphi(t)\left(1-\int_{0}^{t} g(\zeta) \mathrm{d} \zeta\right)\|\nabla u(t)\|^{2} \mathrm{~d} t \\
& \quad+2 \varphi(0)\left(1+\frac{(2 / M)}{1-\int_{0}^{\infty} g(\zeta) \mathrm{d} \zeta}\right) \mathscr{E}_{u}(S),
\end{aligned}
$$


which means that

$$
\begin{aligned}
& \frac{3}{4} \int_{S}^{T} \varphi(t)\left(1-\int_{0}^{t} g(\zeta) \mathrm{d} \zeta\right)\|\nabla u(t)\|^{2} \mathrm{~d} t \\
& \quad \leq \int_{S}^{T} \varphi(t)\left\|u_{t}\right\|^{2} \mathrm{~d} t+\frac{\varepsilon_{1}}{2} \int_{S}^{T} \varphi(t)\|\nabla u(t)\|^{2} \mathrm{~d} t+\frac{1}{k \varepsilon_{1}} \varphi(0) \mathscr{E}_{u}(S)\left(\int_{0}^{\infty} g^{(p-1) / p}(\zeta) \mathrm{d} \zeta\right) \\
& \quad+3 \mathscr{E}_{u}(S) \varphi(0)\left(1+\frac{(2 / M)}{1-\int_{0}^{\infty} g(\zeta) \mathrm{d} \zeta}\right)
\end{aligned}
$$

For simplicity, selecting $\varepsilon_{1}=1-\int_{0}^{\infty} g(\zeta) \mathrm{d} \zeta$, we get

$$
\begin{aligned}
& \int_{S}^{T} \varphi(t)\left(1-\int_{0}^{t} g(\zeta) \mathrm{d} \zeta\right)\|\nabla u(t)\|^{2} \mathrm{~d} t \\
& \quad \leq 4 \int_{S}^{T} \varphi(t)\left\|u_{t}\right\|^{2} \mathrm{~d} t+4\left(\frac{\int_{0}^{\infty} g^{(p-1) / p}(\zeta) \mathrm{d} \zeta}{k\left(1-\int_{0}^{t} g(\zeta) \mathrm{d} \zeta\right)}+3\left(1+\frac{(2 / M)}{1-\int_{0}^{\infty} g(\zeta) \mathrm{d} \zeta}\right)\right) \mathscr{E}_{u}(S) \varphi(0) \\
& \quad \leq 4 \int_{S}^{T} \varphi(t)\left\|u_{t}\right\|^{2} \mathrm{~d} t+\frac{4 M \int_{0}^{\infty} g^{(p-1) / p}(\zeta) \mathrm{d} \zeta+12 k\left(2+M\left(1-\int_{0}^{\infty} g(\zeta) \mathrm{d} \zeta\right)\right)_{\mathscr{E}_{u}}(S) \varphi(0)}{M k\left(1-\int_{0}^{\infty} g(\zeta) \mathrm{d} \zeta\right)} \\
& \quad C_{1} \int_{S}^{T} \varphi(t)\left\|u_{t}\right\|^{2} \mathrm{~d} t+C_{2} \mathscr{E}_{u}(S) \varphi(0),
\end{aligned}
$$

where $C_{1}=4$ and

$$
C_{2}=\frac{4 M \int_{0}^{\infty} g^{(p-1) / p}(\zeta) \mathrm{d} \zeta+12 k\left(2+M\left(1-\int_{0}^{\infty} g(\zeta) \mathrm{d} \zeta\right)\right)}{M k\left(1-\int_{0}^{\infty} g(\zeta) \mathrm{d} \zeta\right)}
$$

are both positive.

Next, multiplying both sides of the original equation (1) by $\varphi(t)$ at the same time, taking $\int_{0}^{t} g(t-\zeta)(u(\zeta)-u(t)) \mathrm{d} \zeta$ as a multiplier, and integrating on the closed interval $[S, T]$, the following equation can be obtained:

$$
\begin{aligned}
& \int_{S}^{T} \varphi(t)\left\langle u_{t t}-\Delta u+\int_{0}^{t} g(t-\zeta) \Delta u(\zeta) \mathrm{d} \zeta-\left(|u|^{\gamma}-\eta^{2}\right) u(t)\right. \\
& \left.\int_{0}^{t} g(t-\zeta)(u(\zeta)-u(t)) \mathrm{d} \zeta\right\rangle \mathrm{d} t=0
\end{aligned}
$$

Taking integration by parts, one obtains

$$
\begin{aligned}
\int_{S}^{T} & \varphi(t)\left\langle u_{t t}, \int_{0}^{t} g(t-\zeta)(u(\zeta)-u(t)) \mathrm{d} \zeta\right\rangle \mathrm{d} t \\
= & \int_{S}^{T} u_{t t} \varphi(t)\left(\int_{0}^{t} g(t-\zeta)(u(\zeta)-u(t)) \mathrm{d} \zeta\right) \mathrm{d} t \\
= & \left.\left(u_{t} \varphi(t) \int_{0}^{t} g(t-\zeta)(u(\zeta)-u(t)) \mathrm{d} \zeta\right)\right|_{S} ^{T}-\int_{S}^{T} u_{t}\left[\varphi(t) \int_{0}^{t} g(t-\zeta)(u(\zeta)-u(t)) d \zeta\right]^{\prime} \mathrm{d} t \\
= & \left.\varphi(t)\left\langle u_{t}, \int_{0}^{t} g(t-\zeta)(u(\zeta)-u(t)) \mathrm{d} \zeta\right\rangle\right|_{S} ^{T}-\int_{S}^{T} \varphi^{\prime}(t)\left\langle u_{t}(t), \int_{0}^{t} g(t-\zeta)(u(\zeta)-u(t)) \mathrm{d} \zeta\right\rangle \mathrm{d} t \\
& \quad-\int_{S}^{T} \varphi(t)\left\langle u_{t}(t), \int_{0}^{t} g^{\prime}(t-\zeta)(u(\zeta)-u(t)) \mathrm{d} \zeta\right\rangle \mathrm{d} t+\int_{S}^{T} \varphi(t)\left\|u_{t}\right\|^{2}\left(\int_{0}^{t} g(\zeta) \mathrm{d} \zeta\right) \mathrm{d} t,
\end{aligned}
$$




$$
\begin{aligned}
\int_{S}^{T} & \varphi(t)\left\langle-\Delta u+\int_{0}^{t} g(t-\zeta) \Delta u(\zeta) \zeta, \int_{0}^{t} g(t-\zeta)(u(\zeta)-u(t)) \mathrm{d} \zeta\right\rangle \mathrm{d} t \\
= & \int_{S}^{T} \varphi(t)\left\langle\nabla u, \int_{0}^{t} g(t-\zeta)(u(\zeta)-u(t)) \mathrm{d} \zeta\right\rangle \mathrm{d} t-\int_{S}^{T} \varphi(t)\left\langle\int_{0}^{t} g(t-\zeta) \nabla u(\zeta) \mathrm{d} \zeta, \int_{0}^{t} g(t-\zeta)(u(\zeta)-u(t)) \mathrm{d} \zeta\right\rangle \mathrm{d} t \\
= & \int_{S}^{T} \varphi(t) \nabla u(t)\left(\int_{0}^{t} g(t-\zeta)(u(\zeta)-u(t)) \mathrm{d} \zeta\right) \mathrm{d} t \\
& \quad-\int_{S}^{T} \varphi(t)\left(\int_{0}^{t} g(t-\zeta)(\nabla u(\zeta)-\nabla u(t)+\nabla u(t)) \mathrm{d} \zeta\right)\left(\int_{0}^{t} g(t-\zeta)(\nabla u(\zeta)-\nabla u(t)) \mathrm{d} \zeta\right) \mathrm{d} t \\
= & \int_{S}^{T} \varphi(t) \nabla u(t)\left(\int_{0}^{t} g(t-\zeta)(u(\zeta)-u(t)) \mathrm{d} \zeta\right) \mathrm{d} t-\int_{S}^{T} \varphi(t)\left(\int_{0}^{t} g(t-\zeta)(\nabla u(\zeta)-\nabla u(t)) \mathrm{d} \zeta\right)^{2} \mathrm{~d} t \\
& \left.\quad-\int_{S}^{T} \varphi(t)\left(\int_{0}^{t} g(t-\zeta) \nabla u(t)\right) \mathrm{d} \zeta\right)\left(\int_{0}^{t} g(t-\zeta)(\nabla u(\zeta)-\nabla u(t)) \mathrm{d} \zeta\right) \mathrm{d} t \\
= & \int_{S}^{T} \varphi(t)\left(1-\int_{0}^{t} g(\zeta) \mathrm{d} \zeta\right)\left\langle\nabla u(t), \int_{0}^{t} g(t-\zeta)(\nabla u(\zeta)-\nabla u(t)) \mathrm{d} \zeta\right\rangle \mathrm{d} t \\
& \quad-\int_{S}^{T} \varphi(t)\left\|\int_{0}^{t} g(t-\zeta)(\nabla u(\zeta)-\nabla u(t)) \mathrm{d} \zeta\right\|^{2} \mathrm{~d} t .
\end{aligned}
$$

Substituting (79) and (80) into (78) leads to the following:

$$
\begin{aligned}
\int_{S}^{T} \varphi & (t)\left(\int_{0}^{t} g(\zeta) \mathrm{d} \zeta\right)\left\|u_{t}\right\|^{2} \mathrm{~d} t \\
= & -\left[\varphi(t)\left\langle u_{t}, \int_{0}^{t} g(t-\zeta)(u(\zeta)-u(t)) \mathrm{d} \zeta\right\rangle\right]_{S}^{T}+\int_{S}^{T} \varphi^{\prime}(t)\left\langle u_{t}(t), \int_{0}^{t} g(t-\zeta)(u(\zeta)-u(t)) \mathrm{d} \zeta\right\rangle \mathrm{d} t \\
& +\int_{S}^{T} \varphi(t)\left\langle u_{t}(t), \int_{0}^{t} g^{\prime}(t-\zeta)(u(\zeta)-u(t)) \mathrm{d} \zeta\right\rangle \mathrm{d} t+\int_{S}^{T} \varphi(t)\left\|\int_{0}^{t} g(t-\zeta)(\nabla u(\zeta)-\nabla u(t)) \mathrm{d} \zeta\right\|^{2} \mathrm{~d} t \\
& +\int_{S}^{T} \varphi(t)\left(-1+\int_{0}^{t} g(\zeta) \mathrm{d} \zeta\right)\left\langle\nabla u(t), \int_{0}^{t} g(t-\zeta)(\nabla u(\zeta)-\nabla u(t)) \mathrm{d} \zeta\right\rangle \mathrm{d} t \\
& +\int_{S}^{T} \varphi(t)\left\langle\left(|u|^{\gamma}-\eta^{2}\right) u(t), \int_{0}^{t} g(t-\zeta)(u(\zeta)-u(t)) \mathrm{d} \zeta\right\rangle \mathrm{d} t .
\end{aligned}
$$

Now, let us consider the term $\int_{S}^{T} \varphi(t)\left\|u_{t}\right\|^{2} \mathrm{~d} t$ and evaluate it. First of all, according to (10), we have

$$
\begin{aligned}
\left|\left\langle u_{t}, \int_{0}^{t} g(t-\zeta)(u(\zeta)-u(t)) \mathrm{d} \zeta\right\rangle\right| & \leq \frac{1}{2}\left[\left\|u_{t}\right\|^{2}+\left(\int_{0}^{t} g(t-\zeta)\|u(\zeta)-u(t)\| \mathrm{d} \zeta\right)^{2}\right] \\
& \leq \mathscr{E}_{u}(t)+\frac{1}{2 M}\left(\int_{0}^{t} g(t-\zeta)\|\nabla u(\zeta)-\nabla u(t)\| \mathrm{d} \zeta\right)^{2} \\
& \leq \mathscr{E}_{u}(t)+\frac{1}{2 M}\left(\int_{0}^{t} g(t-\zeta)\|\nabla u(\zeta)-\nabla u(t)\| \mathrm{d} \zeta\right)\left(\int_{0}^{t} g(\zeta) \mathrm{d} \zeta\right) \\
& \leq \frac{1+M}{M} \mathscr{E}_{u}(t) .
\end{aligned}
$$


Consequently,

$-\left[\varphi(t)\left\langle u_{t}, \int_{0}^{t} g(t-\zeta)(u(\zeta)-u(t)) \mathrm{d} \zeta\right\rangle\right]_{S}^{T} \leq 2 \varphi(0) \frac{1+M}{M} \mathscr{E}_{u}(S)$.

As a result,

$$
\begin{aligned}
& \int_{S}^{T} \varphi^{\prime}(t)\left\langle u_{t}(t), \int_{0}^{t} g(t-\zeta)(u(\zeta)-u(t)) \mathrm{d} \zeta\right\rangle \mathrm{d} t \\
& \quad \leq \frac{1+M}{M} \int_{S}^{T}\left(-\varphi^{\prime}(t)\right) \mathscr{E}_{u}(t) \mathrm{d} t \leq \frac{1+M}{M} \mathscr{E}_{u}(S) \int_{S}^{T}\left(-\varphi^{\prime}(t)\right) \mathrm{d} t \\
& \quad \leq \frac{1+M}{M} \mathscr{E}_{u}(S) \varphi(0) .
\end{aligned}
$$

Using the Cauchy inequality, we have

$$
\begin{aligned}
& \int_{S}^{T} \varphi(t)\left\langle u_{t}(t), \int_{0}^{t} g^{\prime}(t-\zeta)(u(\zeta)-u(t)) \mathrm{d} \zeta\right\rangle \mathrm{d} t \\
& \quad \leq \frac{\delta_{1}}{2} \int_{S}^{T} \varphi(t)\left\|u_{t}\right\|^{2} \mathrm{~d} t+\frac{1}{2 \delta_{1}} \int_{S}^{T} \varphi \\
& \cdot(t)\left(\int_{0}^{t}\left|g^{\prime}(t-\zeta)\right| \cdot \|(u(\zeta)-u(t) \| \mathrm{d} \zeta)^{2} \mathrm{~d} t .\right.
\end{aligned}
$$

Recall that $\left|g^{\prime}(\zeta)\right|=-g^{\prime}(\zeta)$, which is deduced from $g^{\prime}(t) \leq 0$. Hence,

$$
\begin{aligned}
\left(\int_{0}^{t}\left|g^{\prime}(t-\zeta)\right| \cdot \|(u(\zeta)-u(t) \| \mathrm{d} \zeta)^{2}\right. & =\left(\int_{0}^{t}\left(\sqrt{\left|g^{\prime}(t-\zeta)\right|}\right) \cdot\left(\sqrt{\left|g^{\prime}(t-\zeta)\right|} \|(u(\zeta)-u(t) \|) \mathrm{d} \zeta\right)^{2}\right. \\
& \left.\leq-\int_{0}^{t} g^{\prime}(\zeta)\right) \mathrm{d} \zeta\left(\int_{0}^{t}\left|g^{\prime}(t-\zeta)\right| \cdot \|\left(u(\zeta)-u(t) \|^{2} \mathrm{~d} \zeta\right)\right. \\
& =(g(0)-g(t))\left(\int_{0}^{t}\left(-g^{\prime}(t-\zeta)\right) \cdot \|\left(u(\zeta)-u(t) \|^{2} \mathrm{~d} \zeta\right)\right. \\
& \leq g(0)\left(\int_{0}^{t}\left(-g^{\prime}(t-\zeta)\right) \cdot \|\left(u(\zeta)-u(t) \|^{2} \mathrm{~d} \zeta\right)\right. \\
& \leq \frac{g(0)}{M} \int_{0}^{t}\left(-g^{\prime}(t-\zeta)\right) \cdot \|\left(u(\zeta)-u(t) \|^{2} \mathrm{~d} \zeta\right. \\
& \leq-\frac{2 \mathscr{E}_{u}^{\prime}(t) g(0)}{M} .
\end{aligned}
$$

Then, (85) is transformed into the following form:

$$
\begin{aligned}
& \int_{S}^{T} \varphi(t)\left\langle u_{t}(t), \int_{0}^{t} g^{\prime}(t-\zeta)(u(\zeta)-u(t)) \mathrm{d} \zeta\right\rangle \mathrm{d} t \\
& \quad \leq \frac{\delta_{1}}{2} \int_{S}^{T} \varphi(t)\left\|u_{t}\right\|^{2} \mathrm{~d} t+\frac{1}{2 \delta_{1}} \int_{S}^{T} \varphi(t)\left(\int_{0}^{t}\left|g^{\prime}(t-\zeta)\right| \cdot \|(u(\zeta)-u(t) \| \mathrm{d} \zeta)^{2} \mathrm{~d} t\right. \\
& \quad \leq \frac{\delta_{1}}{2} \int_{S}^{T} \varphi(t)\left\|u_{t}\right\|^{2} \mathrm{~d} t+\frac{1}{2 \delta_{1}} \int_{S}^{T} \frac{-2 g(0) \varphi(t) \mathscr{E}_{u}^{\prime}(t)}{M} \mathrm{~d} t \\
& \quad \leq \frac{\delta_{1}}{2} \int_{S}^{T} \varphi(t)\left\|u_{t}\right\|^{2} \mathrm{~d} t+\left.\frac{1}{2 \delta_{1}} \frac{2 g(0) \varphi(0)}{M}\left(-\mathscr{E}_{u}(t)\right)\right|_{S} ^{T} \\
& \quad \leq \frac{\delta_{1}}{2} \int_{S}^{T} \varphi(t)\left\|u_{t}\right\|^{2} \mathrm{~d} t+\frac{g(0) \varphi(0)}{M \delta_{1}} \mathscr{E}_{u}(S) .
\end{aligned}
$$


In view of the assumption of $g(t)$ and $\int_{0}^{\infty} g(\zeta) \mathrm{d} \zeta<1$, the estimates of (64) can be arrived as follows:

$$
\begin{aligned}
& \int_{S}^{T} \varphi(t)\left(-1+\int_{0}^{t} g(\zeta) \mathrm{d} \zeta\right)\left\langle\int_{0}^{t} g(t-\zeta)(\nabla u(\zeta)-\nabla u(t)) \mathrm{d} \zeta, \nabla u(t)\right\rangle \mathrm{d} t \\
& \quad \leq \int_{S}^{T} \varphi(t)\left|\left\langle\int_{0}^{t} g(t-\zeta)(\nabla u(\zeta)-\nabla u(t)) \mathrm{d} \zeta, \nabla u(t)\right\rangle\right| \mathrm{d} t \\
& \quad \leq \frac{\varepsilon_{1}}{2} \int_{S}^{T} \varphi(t)\|\nabla u(t)\|^{2} \mathrm{~d} t+\frac{1}{k \varepsilon_{1}} \varphi(0) \mathscr{E}_{u}(S)\left(\int_{0}^{\infty} g^{(p-1) / p}(\zeta) \mathrm{d} \zeta\right) .
\end{aligned}
$$

Observing that $|F(u)| \leq C\|\nabla u(t)\|^{2+(\gamma / 2)}$, by combining (44) and (45), we can attain that $\forall t \geq 0$,

$$
\begin{aligned}
\|\nabla u(t)\| & \leq \frac{2 \sqrt{\mathscr{E}_{u}(t)}}{\sqrt{1-\int_{0}^{\infty} g(\zeta) \mathrm{d} \zeta}} \leq \frac{2 \sqrt{\mathscr{E}_{u}(0)}}{\sqrt{1-\int_{0}^{\infty} g(\zeta) \mathrm{d} \zeta}} \\
& \leq \frac{2 \rho_{0}}{\sqrt{1-\int_{0}^{\infty} g(\zeta) \mathrm{d} \zeta}}=\sqrt{\frac{2}{\ell}} \rho_{0} .
\end{aligned}
$$

Therefore,

$$
\begin{aligned}
\left\|\left(|u(t)|^{\gamma}-\eta^{2}\right) u(t)\right\| & =\left\|\left(|u(t)|^{\gamma}-\eta^{2}\right) u(t)-\left(|u(0)|^{\gamma}-\eta^{2}\right) u(0)\right\| \\
& \leq C\|\nabla u(t)-\nabla 0\|=C\|\nabla u(t)\| .
\end{aligned}
$$

So, combining (62) with (63) and taking a part of (78) into consideration, we obtain

$$
\begin{aligned}
& \int_{S}^{T} \varphi(t)\left\langle\left(|u|^{\gamma}-\eta^{2}\right) u(t), \int_{0}^{t} g(t-\zeta)(u(\zeta)-u(t)) \mathrm{d} \zeta\right\rangle \mathrm{d} t \\
& \quad \leq C \int_{S}^{T} \varphi(t)\left\langle\left(\|\nabla u(t)\|, \int_{0}^{t} g(t-\zeta)(u(\zeta)-u(t)) \mathrm{d} \zeta\right\rangle \mathrm{d} t\right. \\
& \quad=C \int_{S}^{T} \varphi(t)\left(\|\nabla u(t)\|\left(\int_{0}^{t} g(t-\zeta)(u(\zeta)-u(t)) \mathrm{d} \zeta\right) \mathrm{d} t\right. \\
& \quad \leq \int_{S}^{T} \sqrt{\varphi(t)} \sqrt{\varphi(t)}\left(\|\nabla u(t)\|\left(\int_{0}^{t} g(t-\zeta)(u(\zeta)-u(t)) \mathrm{d} \zeta\right) \mathrm{d} t\right. \\
& \quad=\int_{S}^{T}(\sqrt{\varphi(t)}\|\nabla u(t)\|)\left(\frac{C}{\sqrt{M}} \sqrt{\varphi(t)} \int_{0}^{t} g(t-\zeta)\|\nabla u(\zeta)-\nabla u(t)\| \mathrm{d} \zeta\right) \mathrm{d} t \\
& \quad \leq \frac{\varepsilon_{2}}{2} \int_{S}^{T} \varphi(t)\|\nabla u(t)\|^{2} \mathrm{~d} t+\frac{C^{2}}{M k \varepsilon_{2}} \varphi(0) \mathscr{E}_{u}(S)\left(\int_{0}^{\infty} g^{(p-1) / p}(\zeta) \mathrm{d} \zeta\right) .
\end{aligned}
$$

Since $\int_{0}^{\infty} g(\zeta) \mathrm{d} \zeta<1$, it is natural to see that $\int_{0}^{t} g(\zeta) \mathrm{d} \zeta$ can be regarded as a small number tending to 0 . Now, we consider the existence of such a $t_{0} \in(0, t)$, which guarantees the positiveness of $\int_{0}^{t} g(\zeta) d \zeta$. Further, by a combination of equations (63), (83), (84), (87), (88), and (91), the variant of (81) can be obtained, which satisfies the following estimation:

$$
\begin{aligned}
\int_{S}^{T} \varphi(t) & \left(\int_{0}^{t} g(\zeta) \mathrm{d} \zeta-\frac{\delta}{2}\right)\left\|\nabla u_{t}(t)\right\|^{2} \mathrm{~d} t \\
\leq & \varepsilon_{3} \int_{S}^{T} \varphi(t)\left\|\nabla u_{t}(t)\right\|^{2} \mathrm{~d} t \\
& +\left[\frac{g(0)}{M \delta_{2}}+\frac{3(2 M+1)}{2 M}+\frac{1}{k}\left(2+\frac{1}{\varepsilon_{3}}+\frac{C^{2}}{M \varepsilon_{3}}\right) \int_{0}^{\infty} g^{(p-1) / p}(\zeta) \mathrm{d} \zeta\right] \varphi(0) \mathscr{E}_{u}(S) .
\end{aligned}
$$


For any $S_{0} \in(0, S]$, with the help of the fact $g(0)>0$ and the continuity of $g$, it can be acquired that

$$
\int_{0}^{S} g(\zeta) \mathrm{d} \zeta \geq \int_{0}^{S_{0}} g(\zeta) \mathrm{d} \zeta>0
$$

Choosing a positive constant $\delta_{3}$ which is small enough so that $\delta_{3}<\int_{0}^{S_{0}} g(\zeta) \mathrm{d} \zeta$, and considering

$$
\int_{0}^{t} g(\zeta) \mathrm{d} \zeta<\int_{0}^{S} g(\zeta) \mathrm{d} \zeta
$$

we can check that

$$
\int_{0}^{t} g(\zeta) \mathrm{d} \zeta-\frac{\delta_{3}}{2}<\delta_{3}-\frac{\delta_{3}}{2}=\frac{\delta_{3}}{2} .
$$

Now, for any $S \in\left[S_{0}, T\right)$, we have

$$
\begin{aligned}
& \frac{\delta_{3}}{2} \int_{S}^{T} \varphi(t)\left\|u_{t}(t)\right\|^{2} \mathrm{~d} t \\
& \quad<\frac{1}{2} \int_{0}^{S_{0}} g(\zeta) \mathrm{d} \zeta \int_{S}^{T} \varphi(t)\left\|u_{t}(t)\right\|^{2} \mathrm{~d} t \\
& \quad \leq \varepsilon_{3} \int_{S}^{T} \varphi(t)\left\|\nabla u_{t}(t)\right\|^{2} \mathrm{~d} t+\left[\frac{g(0)}{M \delta_{3}}+\frac{3(2 M+1)}{2 M}+\frac{1}{k}\left(2+\frac{1}{\varepsilon_{3}}+\frac{C^{2}}{M \varepsilon_{3}}\right) \int_{0}^{\infty} g^{(p-1) / p}(\zeta) \mathrm{d} \zeta\right] \varphi(0) \mathscr{E}_{u}(S) \\
& \quad \triangleq \varepsilon_{3} \int_{S}^{T} \varphi(t)\left\|\nabla u_{t}(t)\right\|^{2} \mathrm{~d} t+C_{01} \varphi(0) \mathscr{E}_{u}(S) .
\end{aligned}
$$

Thus, we can conclude that

$$
\int_{S}^{T} \varphi(t)\left\|u_{t}(t)\right\|^{2} \mathrm{~d} t \leq \varepsilon_{4} \int_{S}^{T} \varphi(t)\left\|\nabla u_{t}(t)\right\|^{2} \mathrm{~d} t+C_{1} \varphi(0) \mathscr{E}_{u}(S)
$$

where $\varepsilon_{4}=\left(2 \varepsilon_{3} / \delta_{3}\right)$, and

$C_{1}=\frac{2}{\delta_{3}}\left[\frac{g(0)}{M \delta_{3}}+\frac{3(2 M+1)}{2 M}+\frac{1}{k}\left(2+\frac{1}{\varepsilon_{3}}+\frac{C^{2}}{M \varepsilon_{3}}\right) \int_{0}^{\infty} g^{(p-1) / p}(\zeta) \mathrm{d} \zeta\right]$.

Consequently,

$$
\begin{aligned}
& \int_{S}^{T} \varphi(t)\left(1-\int_{0}^{t} g(\zeta) \mathrm{d} \zeta\right)\|\nabla u(t)\|^{2} \mathrm{~d} t \\
& \quad \leq \mathcal{E}_{5} C_{1} \int_{S}^{T} \varphi(t)\|\nabla u(t)\|^{2} \mathrm{~d} t+C_{2} \varphi(0) \mathscr{E}_{u}(S) .
\end{aligned}
$$

If $\varepsilon_{5}$ is small enough in the above formula, then

$$
\int_{S}^{T} \varphi(t)\left(1-\int_{0}^{t} g(\zeta) \mathrm{d} \zeta\right)\|\nabla u(t)\|^{2} \mathrm{~d} t \leq C_{2} \varphi(0) \mathscr{E}_{u}(S) .
$$

Alternatively, if $C_{3}$ is taken properly, the estimation can be arrived as

$$
\int_{S}^{T} \varphi(t)\left\|u_{t}(t)\right\|^{2} \mathrm{~d} t \leq C_{3} \varphi(0) \mathscr{E}_{u}(S)
$$
get

Considering the third term and the fourth one of (56), we

$$
\begin{aligned}
& -\int_{S}^{T} \varphi(t)\left(\frac{1}{\gamma+2} \int_{\Omega}|u|^{\gamma+2} \mathrm{~d} x-\frac{\eta^{2}}{2} \int_{\Omega}|u|^{2} \mathrm{~d} x\right) \mathrm{d} t \\
& \leq C \int_{S}^{T} \varphi(t)\|\nabla u(t)\|^{2+(\gamma / 2)} \mathrm{d} t \leq \frac{C}{4} \int_{S}^{T} \varphi(t) \\
& \quad \cdot\left(1-\int_{0}^{\infty} g(\zeta) \mathrm{d} \zeta\right)\|\nabla u(t)\|^{2} \mathrm{~d} t \\
& \leq \frac{C}{4} \int_{S}^{T} \varphi(t)\left(1-\int_{0}^{t} g(\zeta) \mathrm{d} \zeta\right)\|\nabla u(t)\|^{2} \mathrm{~d} t \leq C_{4} \varphi(0) \mathscr{E}_{u}(S) .
\end{aligned}
$$

By combining equations (100)-(102), it is shown that (56) is true, which concludes the proof.

Next, it remains to complete the Proof of Theorem 2.

Proof of Theorem 2. Let us consider the case where $p$ equals infinity firstly. For any $t$, if $c$ represents any positive constant, then by taking $\varphi(t)=c$ in (56), we can get the following result:

$$
\begin{aligned}
& \frac{1}{2} \int_{S}^{T}\left\|u_{t}\right\|^{2} \mathrm{~d} t+\frac{1}{2} \int_{S}^{T}\left(1-\int_{0}^{t} g(\zeta) \mathrm{d} \zeta\right)\|\nabla u(t)\|^{2} \mathrm{~d} t \\
& -\frac{1}{\gamma+2} \int_{S}^{T} \int_{\Omega}|u|^{\gamma+2} \mathrm{~d} x \mathrm{~d} t+\frac{\eta^{2}}{2} \int_{S}^{T} \int_{\Omega}|u|^{2} \mathrm{~d} x \mathrm{~d} t \\
& \leq C_{5} \mathscr{E}_{u}(S) .
\end{aligned}
$$


Again, $g(t) \leq-\left(g^{\prime}(t) / k\right)$ follows from $g^{\prime}(t) \leq-k g(t)$. Invoking Lemma 3, one may deduce that

$$
\begin{aligned}
& \frac{1}{2} \int_{S}^{T}\left(\int_{0}^{t} g(t-\zeta)\|\nabla u(\zeta)-\nabla u(t)\|^{2} \mathrm{~d} \zeta\right) \mathrm{d} t \\
& \quad \leq-\frac{1}{k} \frac{1}{2} \int_{S}^{T}\left(\int_{0}^{t} g^{\prime}(t-\zeta)\|\nabla u(\zeta)-\nabla u(t)\|^{2} \mathrm{~d} \zeta\right) \mathrm{d} t \\
& \quad \leq-\frac{1}{k} \int_{S}^{T} \mathscr{E}_{u}^{\prime}(t) \mathrm{d} t \\
& \quad \leq \frac{1}{k} \mathscr{E}_{u}(S) .
\end{aligned}
$$

Then, $\int_{S}^{T} \mathscr{E}_{u}^{(p+1 / p)}(t) \mathrm{d} t \leq C \mathscr{E}_{u}(S)$ can be derived from (55). This fact further explains the attenuation of $\mathscr{E}_{u}(t)$ according to a polynomial form.
Secondly, it is valuable to consider the case of $2<p<\infty$. Aiming to evaluate the last term of $\mathscr{E}_{u}(t)$, we will put forward the following lemmas.

Lemma 5. For any $0 \leq S \leq T$ and $t>0$, the following inequality holds:

$$
\begin{aligned}
& \int_{S}^{T} \varepsilon_{u}^{(1 / p)}(t)\left(\int_{0}^{t} g(t-\zeta)\|\nabla u(\zeta)-\nabla u(t)\|^{2} \mathrm{~d} \zeta\right) \mathrm{d} t \\
& \quad \leq C_{6} \varepsilon_{u}^{p /(p+1)}(S)\left[\int_{S}^{T} \varepsilon_{u}^{(p+1 / p)}(t)\left(\int_{0}^{t}\|\nabla u(\zeta)-\nabla u(t)\|^{2} \mathrm{~d} \zeta\right) \mathrm{d} t\right]^{(1 / p+1)} .
\end{aligned}
$$

Proof. Let

$$
\Phi_{1}(t):=\int_{0}^{t}\|\nabla u(\zeta)-\nabla u(t)\|^{2} \mathrm{~d} \zeta .
$$

In view of the assumption $H_{1}$ and the Hölder inequality, we get

$$
\begin{aligned}
& \int_{0}^{T} g(t-\zeta)\|\nabla u(\zeta)-\nabla u(t)\|^{2} \mathrm{~d} \zeta \\
& \quad=\int_{0}^{T}\left(\|\nabla u(\zeta)-\nabla u(t)\|^{2}\right)^{(1 / p+1)}\left(g^{(p+1 / p)}(t-\zeta)\|\nabla u(\zeta)-\nabla u(t)\|^{2}\right)^{(p / p+1)} \mathrm{d} \zeta \\
& \quad \leq\left[\int_{0}^{T}\left(\|\nabla u(\zeta)-\nabla u(t)\|^{2}\right)^{(1 / p+1)(p+1)} \mathrm{d} \zeta\right]^{(1 / p+1)} \times\left[\int_{0}^{T}\left(g^{(p+1 / p)}(t-\zeta)\|\nabla u(\zeta)-\nabla u(t)\|^{2}\right)^{(p / p+1)(p+1 / p)} \mathrm{d} \zeta\right]^{(p / p+1)} \\
& \quad=\Phi_{1}^{p /(p+1)}(t)\left[\int_{0}^{T} g^{(p+1 / p)}(t-\zeta)\|\nabla u(\zeta)-\nabla u(t)\|^{2} \mathrm{~d} \zeta\right]^{(p / p+1)}
\end{aligned}
$$

Hence,

$$
\begin{aligned}
& \int_{S}^{T} \varepsilon_{u}^{(1 / p)}(t) \int_{0}^{T} g(t-\zeta)\|\nabla u(\zeta)-\nabla u(t)\|^{2} \mathrm{~d} \zeta \mathrm{d} t \\
& \quad \leq \int_{S}^{T} \varepsilon_{u}^{(1 / p)}(t) \Phi_{1}^{(1 / p+1)}(t)\left(\int_{0}^{T} g^{1+(1 / p)}(t-\zeta)\|\nabla u(\zeta)-\nabla u(t)\|^{2} \mathrm{~d} \zeta\right)^{(p / p+1)} \mathrm{d} t \\
& \quad=\int_{S}^{T}\left(\varepsilon_{u}^{(p+1 / p)}(t) \Phi_{1}(t)\right)^{(1 / p+1)}\left(\int_{0}^{T} g^{1+(1 / p)}(t-\zeta)\|\nabla u(\zeta)-\nabla u(t)\|^{2} \mathrm{~d} \zeta\right)^{(p / p+1)} \mathrm{d} t \\
& \quad \leq\left(\int_{S}^{T} \varepsilon_{u}^{(p+1 / p)}(t) \Phi_{1}(t) \mathrm{d} t\right)^{(1 / p+1)}\left(\int_{S}^{T} \int_{0}^{T} g^{1+(1 / p)}(t-\zeta)\|\nabla u(\zeta)-\nabla u(t)\|^{2} \mathrm{~d} \zeta \mathrm{d} t\right)^{(p / p+1)} \\
& \quad \leq\left(\int_{S}^{T} \varepsilon_{u}^{(p+1 / p)}(t) \Phi_{1}(t) \mathrm{d} t\right)^{(1 / p+1)}\left(\int_{S}^{T} \int_{0}^{T}\left(-\frac{g^{\prime}(t-\zeta)}{k}\right)\|\nabla u(\zeta)-\nabla u(t)\|^{2} \mathrm{~d} \zeta \mathrm{d} t\right)^{(p / p+1)}
\end{aligned}
$$




$$
\begin{aligned}
& =k^{-(p / p+1)}\left(\int_{S}^{T} \varepsilon_{u}^{(p+1 / p)}(t) \Phi_{1}(t) \mathrm{d} t\right)^{(1 / p+1)}\left(\int_{S}^{T} \int_{0}^{T}\left(-g^{\prime}(t-\zeta)\right)\|\nabla u(\zeta)-\nabla u(t)\|^{2} \mathrm{~d} \zeta \mathrm{d} t\right)^{(p / p+1)} \\
& \leq k^{-(p / p+1)}\left(\int_{S}^{T} \varepsilon_{u}^{(p+1 / p)}(t) \Phi_{1}(t) \mathrm{d} t\right)^{(1 / p+1)}\left(-2 \int_{S}^{T} \varepsilon_{u}^{\prime}(t) \mathrm{d} t\right)^{(p / p+1)} \\
& =\left(\frac{k}{2}\right)^{-(p / p+1)}\left(\int_{S}^{T} \varepsilon_{u}^{(p+1 / p)}(t) \Phi_{1}(t) \mathrm{d} t\right)^{(1 / p+1)}\left(\varepsilon_{u}(S)-\varepsilon_{u}(T)\right)^{(p / p+1)} \\
& \leq\left(\frac{k}{2}\right)^{-(p / p+1)}\left(\int_{S}^{T} \varepsilon_{u}^{(p+1 / p)}(t) \Phi_{1}(t) \mathrm{d} t\right)^{(1 / p+1)} \varepsilon_{u}^{(p / p+1)}(S) \\
& =C_{6} \varepsilon_{u}^{p /(p+1)}(S)\left(\int_{S}^{T} \varepsilon_{u}^{(p+1 / p)}(t) \Phi_{1}(t) \mathrm{d} t\right)^{(1 / p+1)}
\end{aligned}
$$

where $C_{6}=(k / 2)^{-p /(p+1)}$. This completes the proof.

Lemma 6. Let

$\Phi_{2}(t):=\int_{0}^{t} \sqrt{g(t-\zeta)}\|\nabla u(\zeta)-\nabla u(t)\|^{2} \mathrm{~d} \zeta, \quad \forall t \leq 0$.

Then, for $0 \leq S \leq T$, it holds that

$$
\begin{gathered}
\int_{S}^{T} \varepsilon_{u}^{(2 / p)}(t)\left(\int_{0}^{t} g(t-\zeta)\|\nabla u(\zeta)-\nabla u(t)\|^{2} \mathrm{~d} \zeta\right) \mathrm{d} t \\
\quad \leq C_{7} \varepsilon_{u}^{p /(p+2)}(S)\left[\int_{S}^{T} \varepsilon_{u}^{1+(2 / p)}(t) \Phi_{2}(t) \mathrm{d} t\right]^{(2 / p+2)} .
\end{gathered}
$$

The proof of Lemma 6 can be deduced similar to the one of Lemma 5 , and this process will not be stated here. Besides, it is easy to see that $\Phi_{2}(t)$ is bounded.

Indeed, $\sqrt{g(\zeta)} \in L^{1}(0, \infty)$ can be ensured from $p>2$. With the assistance of (45), we find

$$
\|\nabla u(t)\|^{2} \leq \frac{4 \mathscr{C}_{u}(t)}{1-\int_{0}^{\infty} g(\zeta) \mathrm{d} \zeta},
$$

and

$$
\begin{aligned}
\left|\Phi_{2}(t)\right| \leq C_{2_{a}} \int_{0}^{t} \sqrt{g(t-\zeta)}\left(\|\nabla u(\zeta)\|^{2}+\|\nabla u(t)\|^{2}\right) \mathrm{d} \zeta \\
\leq C_{2_{b}} \int_{0}^{t} \sqrt{g(t-\zeta)}\left(\mathscr{E}_{u}(\zeta)+\mathscr{E}_{u}(t)\right) \mathrm{d} \zeta \\
\leq C_{2_{c}} \int_{0}^{t} \sqrt{g(t-\zeta)} \mathscr{C}_{u}(0) \mathrm{d} \zeta \\
\leq C_{2_{c}} \int_{0}^{\infty} \sqrt{g(t-\zeta)} \mathscr{E}_{u}(0) \mathrm{d} \zeta \\
\leq C_{2_{d}} \int_{0}^{\infty} \sqrt{g(t-\zeta)} \mathscr{E}_{u}(0) \mathrm{d} \zeta \leq C_{2_{e}} \mathscr{E}_{u}(0)
\end{aligned}
$$

where $C_{2_{e}} \geq C_{2_{d}} \int_{0}^{\infty} \sqrt{g(t-\zeta)} d \zeta$. Now,

$$
\left\|\Phi_{2}(t)\right\|_{\infty}^{(2 / p)} \leq C_{2_{e}}^{(2 / p)} \mathscr{E}_{u}(0)^{(2 / p)},
$$

which follows from (112).
Lemma 7. For any $S_{0} \in(0, \infty)$, if $S \geq S_{0}$, then there is a constant $C_{8} \in(0, \infty)$ such that

$$
\int_{S}^{\infty} \varepsilon_{u}^{(p+2 / p)}(t) \mathrm{d} t \leq C_{8} \varepsilon_{u}(S)\left[\varepsilon_{u}^{(2 / p)}(0)+\left\|\Phi_{2}(t)\right\|_{\infty}^{(2 / p)}\right] .
$$


Proof. Given $S \in\left(S_{0}, T\right)$. By means of (39), we get

$$
\begin{aligned}
& \int_{S}^{T} \mathscr{E}_{u}^{(p+2) / p}(t) \mathrm{d} t \\
&=\int_{S}^{T} \mathscr{E}_{u}^{(2 / p)}(t) \mathscr{E}_{u}(t) \mathrm{d} t \\
&=\int_{S}^{T} \mathscr{E}_{u}^{(2 / p)}(t)\left[\frac{1}{2}\left(\left\|u_{t}\right\|^{2}+\left(1-\int_{0}^{t} g(\zeta) \mathrm{d} \zeta\right)\|\nabla u\|^{2}+\int_{0}^{t} g(t-\zeta)\|\nabla u(\zeta)-\nabla u(t)\|^{2} \mathrm{~d} \zeta\right)-\frac{1}{\gamma+2} \int_{\Omega}|u|^{\gamma+2} \mathrm{~d} x+\frac{\eta^{2}}{2} \int_{\Omega}|u|^{2} \mathrm{~d} x\right] \mathrm{d} t \\
&=\frac{1}{2} \int_{S}^{T} \mathscr{E}_{u}^{(2 / p)}(t)\left\|u_{t}\right\|^{2} \mathrm{~d} t+\frac{1}{2} \int_{S}^{T} \mathscr{E}_{u}^{(2 / p)}(t)\left(1-\int_{0}^{t} g(\zeta) \mathrm{d} \zeta\right)\|\nabla u\|^{2} \mathrm{~d} t-\frac{1}{\gamma+2} \int_{S}^{T} \mathscr{E}_{u}^{(2 / p)}(t) \int_{\Omega}|u|^{\gamma+2} \mathrm{~d} x \mathrm{~d} t \\
& \quad+\frac{\eta^{2}}{2} \int_{S}^{T} \mathscr{E}_{u}^{(2 / p)}(t) \int_{\Omega}|u|^{2} \mathrm{~d} x \mathrm{~d} t+\frac{1}{2} \int_{S}^{T} \mathscr{E}_{u}^{(2 / p)}(t) \int_{0}^{t} g(t-\zeta)\|\nabla u(\zeta)-\nabla u(t)\|^{2} \mathrm{~d} \zeta \mathrm{d} t \\
& \triangleq I+I I+I I I+I V+V .
\end{aligned}
$$

$$
I+I I+I I I+I V \leq C \varepsilon_{u}^{(2 / p)}(0) \varepsilon_{u}(S) .
$$

Taking $\varepsilon_{u}^{(2 / p)}(t)$ as a multiplier and replacing the position of $\varphi(t)$ in (56), we have

$$
\begin{aligned}
V & \leq C_{7} \varepsilon_{u}^{p /(p+2)}(S)\left[\int_{S}^{T} \varepsilon_{u}^{1+(2 / p)}(t) \Phi_{2}(t) \mathrm{d} t\right]^{2 /(p+2)} \\
& \leq C_{7} \varepsilon_{u}^{p /(p+2)}(S)\left(\int_{S}^{T} \varepsilon_{u}^{1+(2 / p)}(t) \mathrm{d} t\right)^{2 /(p+2)}\left(\int_{S}^{T} \Phi_{2}(t) \mathrm{d} t\right)^{2 /(p+2)} \\
& \leq C_{7} \varepsilon_{u}^{p /(p+2)}(S)\left(\int_{S}^{T} \varepsilon_{u}^{1+(2 / p)}(t) \mathrm{d} t\right)^{(2 / p+2)}\left\|\Phi_{2}(t)\right\|_{\infty}^{2 /(p+2)} \\
& \leq \varepsilon_{6}\left(\int_{S}^{T} \varepsilon_{u}^{1+(2 / p)}(t) \mathrm{d} t\right)^{(2 /(p+2) \cdot(p+2) / 2)}+\frac{\left(2 \varepsilon_{6} / p+2\right)^{(-p / 2)}}{(p / p+2)}\left(\varepsilon_{u}^{p /(p+2)}(S)\right)^{(p+2) / p}\left\|\Phi_{2}(t)\right\|_{\infty}^{(2 /(p+2) \cdot(p+2) / p)} \\
& =\varepsilon_{6} \int_{S}^{T} \varepsilon_{u}^{1+(2 / p)}(t) \mathrm{d} t+\frac{\left(2 \varepsilon_{6} / p+2\right)^{(-p / 2)}}{(p / p+2)} \varepsilon_{u}(S)\left\|\Phi_{2}(t)\right\|_{\infty}^{(2 / p)} .
\end{aligned}
$$

Combining (116) and (117), one gets

$$
\int_{S}^{T} \varepsilon_{u}^{(p+2) / p}(t) \mathrm{d} t \leq \varepsilon_{6} \int_{S}^{T} \varepsilon_{u}^{1+(2 / p)}(t) \mathrm{d} t+\varepsilon_{u}(S)\left(\frac{\left(2 \varepsilon_{6} / p+2\right)^{(-p / 2)}}{(p / p+2)}\left\|\Phi_{2}(t)\right\|_{\infty}^{(2 / p)}+C \varepsilon_{u}^{(2 / p)}(0)\right)
$$

Let the positive number $\varepsilon_{6}$ be infinitely close to zero, and

$$
\int_{S}^{T} \varepsilon_{u}^{(p+2) / p}(t) \mathrm{d} t \leq C_{8} \varepsilon_{u}(S)\left(\left\|\Phi_{2}(t)\right\|_{\infty}^{(2 / p)}+\varepsilon_{u}^{(2 / p)}(0)\right) .
$$


As $T$ tends to infinity, the limit result of long-time memory is easily seen, and thus, (114) is true.

Remark 3. With the aid of paper [12], it is trivial to show that

$$
\int_{S}^{\infty} \varepsilon_{u}^{(p+1) / p}(t) \mathrm{d} t \leq C_{8^{\prime}} \varepsilon_{u}(S)\left[\varepsilon_{u}^{(1 / p)}(0)+\left\|\Phi_{1}(t)\right\|_{\infty}^{(1 / p)}\right] .
$$

The proof of (120) is entirely similar to that of (114) and so it is omitted here. Now, let us turn back to complete the verification of Theorem 2 . have

Continued Proof of Theorem 2. By equation (113), we

$$
\begin{aligned}
\int_{S}^{\infty} \varepsilon_{u}^{(p+2) / p}(t) \mathrm{d} t & \leq C_{8} \varepsilon_{u}(S)\left[\varepsilon_{u}^{(2 / p)}(0)+C_{2_{e}}^{(2 / p)} \varepsilon_{u}(0)^{(2 / p)}\right] \\
& \leq C_{9} \varepsilon_{u}(S) \varepsilon_{u}^{(2 / p)}(0),
\end{aligned}
$$

where $C_{9} \geq C_{8}\left(1+C_{2_{e}}^{(2 / p)}\right)$.

Taking $(2 / p)<1, T=S$ and $T_{0}=S_{0}$, it is inferred from (121) that

$$
\mathscr{E}_{u}(t) \leq \mathscr{E}_{u}(0)\left[\frac{\left(S_{0}+C\right)(1+(2 / p))}{(2 t / p)+S_{0}+C}\right]^{(p / 2)}, \quad \forall t \in[0,+\infty) .
$$

From the representation of (45), it is not difficult to examine that for any $t \in[0, \infty)$,

$$
\begin{aligned}
\left|\Phi_{1}(t)\right| & =\left|\int_{0}^{t}\|\nabla u(\zeta)-\nabla u(t)\|^{2} \mathrm{~d} \zeta\right| \leq C_{10}\left(\int_{0}^{t} \mathscr{E}_{u}(\zeta) \mathrm{d} \zeta+\int_{0}^{t} \mathscr{E}_{u}(t) \mathrm{d} t\right) \\
& \leq C_{10}\left(\int_{0}^{\infty} \mathscr{E}_{u}(\zeta) \mathrm{d} \zeta+t \mathscr{E}_{u}(t)\right) \leq C_{10}\left(\int_{0}^{t} \mathscr{E}_{u}(\zeta) \mathrm{d} \zeta+C_{11} t \mathscr{E}_{u}(0)\right) \\
& \leq C_{12} \mathscr{E}_{u}(0),
\end{aligned}
$$

where $C_{12} \geq t\left(1+C_{11}\right) C_{10}$. That is, $\left\|\Phi_{1}(t)\right\|_{\infty} \leq C_{12} \mathscr{E}_{u}(0)$.

The application of (120) and $S \geq S_{0}$ yields

$$
\int_{S}^{\infty} \varepsilon_{u}^{(p+1) / p}(t) \mathrm{d} t \leq C_{13} \varepsilon_{u}(S) \varepsilon_{u}^{(1 / p)}(0) .
$$

Thus, employing $p>2$, we have

$$
\mathscr{E}_{u}(t) \leq \mathscr{E}_{u}(0)\left[\frac{\left(S_{0}+C\right)(1+(1 / p))}{(t / p)+S_{0}+C}\right]^{p}=\mathscr{E}_{u}(0)\left(\frac{p+1}{p+C t}\right)^{p}
$$

Besides, under the condition that $p=\infty$, equation (124) turns into

$$
\int_{S}^{\infty} \mathscr{E}_{u}(t) \mathrm{d} t \leq C_{13} \mathscr{E}_{u}(S)
$$

which yields

$$
\mathscr{E}_{u}(t) \leq \mathscr{E}_{u}(0) e^{1-\left(t / C_{13^{\prime}}\right)}=\mathscr{E}_{u}(0) e^{1-C t}
$$

This completes the proof.

\section{Conclusion}

Based on the proposed appropriate assumptions of the convolution kernels along with the discussion about the fuzzy number $\eta$, the exponential and polynomial aspects of the energy decay rates for system (1) are estimated only through the application of the multiplier method and the unified technique. In this process, the most valuable point is that our research has avoided the construction of auxiliary functions perfectly. The appearance of the term with fuzzy coefficient makes the expression form of $\mathscr{E}_{u}$ richer and it leads to some difficulties in calculation. At the same time, more efforts have been spent on discussing the integro-differential inequalities and the discussion is quite interesting. Considering the case of $\eta=0$, we can see that the results coincide with that of reference [12]. In summary, the result in this paper reveals the wide applicability of the unified method, and further discussion for the blow-up problems may be considered in the future.

\section{Data Availability}

All datasets generated for this study are included in the manuscript.

\section{Conflicts of Interest}

The authors declare that they have no conflicts of interest.

\section{Acknowledgments}

This study was funded by the Youth Foundation of Jining University (Grant no. 2019QNKJ03), Guangxi Natural Science Foundation (Grant no. 2020GXNSFAA297010), and the Basic Ability Promotion Project for Young and Middleaged Teachers of Guangxi Colleges and Universities (Grant 
no. 2021KY0651, 2019KY0669). The authors are grateful to Professor Fushan Li for his helpful discussions and insightful comments.

\section{References}

[1] M. M. Cavalcanti and H. P. Oquendo, "Frictional versus viscoelastic damping in a semilinear wave equation," SIAM Journal on Control and Optimization, vol. 42, no. 4, pp. 1310-1324, 2003.

[2] S. A. Messaoudi and N.-e. Tatar, "Exponential and polynomial decay for a quasilinear viscoelastic equation," Nonlinear Analysis: Theory, Methods \& Applications, vol. 68, no. 4, pp. 785-793, 2008.

[3] S. Berrimi and S. A. Messaoudi, "Existence and decay of solutions of a viscoelastic equation with a nonlinear source," Nonlinear Analysis: Theory, Methods \& Applications, vol. 64, no. 10, pp. 2314-2331, 2006.

[4] N.-E. Tatar, "Exponential decay for a viscoelastic problem with a singular kernel," Zeitschrift für angewandte Mathematik und Physik, vol. 60, no. 4, pp. 640-650, 2009.

[5] F. Mesloub and S. Boulaaras, "General decay for a viscoelastic problem with not necessarily decreasing kernel," Journal of Applied Mathematics and Computing, vol. 58, no. 1-2, pp. 647-665, 2018.

[6] F. Li and C. Zhao, "Uniform energy decay rates for nonlinear viscoelastic wave equation with nonlocal boundary damping," Nonlinear Analysis: Theory, Methods \& Applications, vol. 74, no. 11, pp. 3468-3477, 2011.

[7] S. A. Messaoudi and W. Khulaifi, "General and optimal decay for a quasilinear viscoelastic equation," Applied Mathematics Letters, vol. 66, pp. 16-22, 2016.

[8] F. S. Li and Z. Q. Jia, "Global existence and stability of a class of nonlinear evolution equations with hereditary memory and variable density," Bound Value Problems, vol. 37, 2019.

[9] F. Q. Li and M. Nadeem, "He-Laplace method for nonlinear vibration in shallow water waves," Journal of Low Frequency Noise, Vibration and Active Control, vol. 38, no. 3-4, pp. 1305-1313, 2019.

[10] H. Liu, S. Li, H. Wang, and G. Li, "Adaptive fuzzy synchronization for a class of fractional-order neural networks," Chinese Physics B, vol. 26, no. 3, Article ID 030504, 2017.

[11] H. Liu, Y. Pan, S. Li, and Y. Chen, "Adaptive fuzzy backstepping control of fractional-order nonlinear systems," IEEE Transactions on Systems, Man, and Cybernetics: Systems, vol. 47, no. 8, pp. 2209-2217, 2017.

[12] F. Alabau-Boussouira, P. Cannarsa, and D. Sforza, "Decay estimates for second order evolution equations with memory," Journal of Functional Analysis, vol. 254, no. 5, pp. 1342-1372, 2008.

[13] D. Xu, "Observability inequalities for Hermite Bi-cubic orthogonal spline collocation methods of 2-D integro-differential equations in the square domains," Applied Mathematics \& Optimization, vol. 2020, 2020.

[14] A. Lorenzi, "Linear integro-differential schrödinger and plate problems without initial conditions," Applied Mathematics \& Optimization, vol. 67, no. 3, pp. 391-418, 2013.

[15] L. C. Evans, "Partial differential equations," in Proceedings of the Graduate Studies in Mathematics, American Mathematical Society, Providence, RI, USA, 1998. 